\title{
Cloud hybrid methods for solving split equilibrium and fixed point problems for a family of countable quasi-Lipschitz mappings and applications
}

\author{
Yongchun $\mathrm{Xu}^{\mathrm{a}}$, Yanxia Tang ${ }^{\mathrm{a}}$, Jinyu Guan ${ }^{\mathrm{b}}$, Yongfu Su ${ }^{\mathrm{b}, *}$ \\ ${ }^{a}$ Department of Mathematics, College of Science, Hebei North University, Zhangjiakou 075000, China. \\ ${ }^{b}$ Department of Mathematics, Tianjin Polytechnic University, Tianjin 300387, China.
}

Communicated by X. Qin

\begin{abstract}
The purpose of this article is to introduce a new multidirectional hybrid shrinking projection iterative algorithm (or called cloud hybrid shrinking projection iterative algorithm) for solving the common element problems which consist of a generalized split equilibrium problems and fixed point problems for a family of countable quasi-Lipschitz mappings in the framework of Hilbert spaces. It is proved that under appropriate conditions, the sequence generated by the multidirectional hybrid shrinking projection method, converges strongly to some point which is the common fixed point of a family of countable quasi-Lipschitz mappings and the solution of the generalized split equilibrium problems. This iteration algorithm can accelerate the convergence speed of iterative sequence. The main results were also applied to solve split variational inequality problem and split optimization problems. Meanwhile, the main results were also used for solving common problems which consist of a generalized split equilibrium problems and fixed point problems for asymptotically nonexpansive mappings. The results of this paper improve and extend the previous results given in the literature. (C)2017 All rights reserved.
\end{abstract}

Keywords: Hybrid shrinking projection, split equilibrium problem, fixed point, quasi-Lipschitz mapping, split variational inequality, split optimization problem.

2010 MSC: 47H05, 47H09, 47H10.

\section{Introduction}

In 1994, Blum and Oettli [2] introduced the equilibrium problem which is to find $x \in C$ such that

$$
\mathrm{F}(\mathrm{x}, \mathrm{y}) \geqslant 0, \quad \forall \mathrm{y} \in \mathrm{C} .
$$

They denoted the solution set of problem (1.1) as EP(F). Since the well-known problems were variational problems, complementary problems, fixed point problems, saddle point problems and other problems proposed from the equilibrium problem, it has become the most attractive topic for many mathematicians

\footnotetext{
*Corresponding author

Email addresses: hbxuyongchun@163.com (Yongchun Xu), sutang2016@163.com (Yanxia Tang), guanjinyu2010@163.com (Jinyu Guan), t j suyongfu@163. com (Yongfu Su)
}

doi:10.22436/jnsa.010.02.36 
$[12,15-17]$. They have widely spread its applications to other applied disciplines including physics, chemistry, economics and engineering (see, for example, [5, 13, 19, 20]).

Combettes and Hirstoaga [7] proposed an iterative method for solving problem (1.1) by the assumption that $\operatorname{EP}(F) \neq \emptyset$. Moreover, there are many new iteratively generated sequences for solving this problem together with fixed point problems (see [1, 8, 21-23]).

Later, the so-called split equilibrium problem was introduced (shortly, SEP). Let $\mathrm{H}_{1}, \mathrm{H}_{2}$ be two real Hilbert spaces. Let $C, Q$ be closed convex subsets of $H_{1}$ and $H_{2}$, respectively, and let $A: H_{1} \rightarrow H_{2}$ be a bounded linear operator. Further, let $F_{1}: C \times C \rightarrow R$ and $F_{2}: Q \times Q \rightarrow R$ be two bifunctions. The SEP is to find the element $x^{*} \in C$ such that

$$
F_{1}\left(x^{*}, y\right) \geqslant 0, \quad \forall y \in C,
$$

and such that

$$
A x^{*} \in Q \text { solves } F_{2}\left(A x^{*}, v\right) \geqslant 0, \quad \forall v \in Q .
$$

The solution sets of problems (1.2) and (1.3) are symbolized by $\operatorname{EP}\left(F_{1}\right)$ and $\operatorname{EP}\left(F_{2}\right)$, respectively. Therefore, we denote $\Omega=\left\{v \in C: v \in \operatorname{EP}\left(F_{1}\right)\right.$ such that $\left.A v \in E P\left(F_{2}\right)\right\}$ as the solution set of SEP.

Clearly, the SEP contains two equilibrium problems, that is, we find out the solution of one equilibrium problem, i.e., its image under a given bounded linear operator must be the solution of another equilibrium problem. In order to find a common solution of equilibrium problems, it has been mostly considered in the same spaces. However, we normally found that, in the real-life problems, it may be considered in different spaces. That is how the SEP works very well for this case (see, for example, [10]). Moreover, the split variational inequality problem (shortly, SVIP) is its special case which is to find $x^{*} \in \mathrm{C}$ such that

$$
\left\langle f\left(x^{*}\right), x-x^{*}\right\rangle \geqslant 0, \quad \forall x \in C,
$$

and corresponding to

$$
\mathrm{y}^{*}=A x^{*} \in Q \text { solves }\left\langle g\left(y^{*}\right), y-y^{*}\right\rangle \geqslant 0, \quad \forall y \in Q,
$$

where $f: H_{1} \rightarrow H_{2}$ and $g: H_{1} \rightarrow H_{2}$ are nonlinear mappings and $A: H_{1} \rightarrow H_{2}$ is a bounded linear operator (see [4]).

In 2012, He [10] proposed the new algorithm for solving a split equilibrium problem and investigated the convergence behavior in several ways including both weak and strong convergence. Moreover, they gave some examples and mentioned that there exist many SEPs and the new methods for solving it further need to be explored in the future. Later, in 2013, Kazmi and Rizvi [14] considered the iterative method to compute the common approximate solution of a split equilibrium problem, a variational inequality problem and a fixed point problem for a nonexpansive mapping in the framework of real Hilbert spaces. They generated the sequence iteratively as follows:

$$
\left\{\begin{array}{l}
u_{n}=J_{r_{n}}^{F_{1}}\left(I+\gamma A^{*}\left(J_{r_{n}}^{F_{2}}-I\right) A\right) x_{n} \\
y_{n}=P_{C}\left(u_{n}-\lambda_{n} D u_{n}\right) \\
x_{n+1}=\alpha_{n} v+\beta_{n} x_{n}+\gamma_{n} S y_{n}
\end{array}\right.
$$

for each $n \geqslant 0$, where $A: H_{1} \rightarrow H_{2}$ is a bounded linear operator, $D: C \rightarrow H_{1}$ is a $\tau$-inverse strongly monotone mapping, $\mathrm{F}_{1}: \mathrm{C} \times \mathrm{C} \rightarrow \mathrm{R}, \mathrm{F}_{2}: \mathrm{Q} \times \mathrm{Q} \rightarrow \mathrm{R}$ are two bifunctions. They found that, under the sufficient conditions of $r_{n}, \lambda_{n}, \gamma, \beta_{n}$ and $\gamma_{n}$, the generated sequence $\left\{x_{n}\right\}$ converges strongly to a common solution of all mentioned problems.

Recently, in 2014, Bnouhachem [3] introduced a new iterative method for solving split equilibrium problem and hierarchical fixed point problems by defining the sequence $\left\{x_{n}\right\}$ as follows:

$$
\left\{\begin{array}{l}
u_{n}=T_{r_{n}}^{F_{1}}\left(I+\gamma A^{*}\left(T_{r_{n}}^{F_{2}}-I\right) A\right) x_{n} \\
y_{n}=\beta_{n} S x_{n}+\left(1-\beta_{n}\right) u_{n} \\
x_{n+1}=P_{C}\left[\alpha_{n} \rho U\left(x_{n}\right)+\left(I-\alpha_{n} \mu F\right)\left(T\left(y_{n}\right)\right)\right]
\end{array}\right.
$$


for each $n \geqslant 0$, where $S, T$ are nonexpansive mappings, $F: C \rightarrow C$ is a $k$-Lipschitz mapping and $\eta$-strongly monotone, $\mathrm{U}: \mathrm{C} \rightarrow \mathrm{C}$ is a $\tau$-Lipschitz mapping. Also, they proved some strong convergence theorems for the proposed iteration under some appropriate conditions.

In 2015 [24], motivated and inspired by the results [3, 10, 14] and the recent works in this field, Witthayarat et al. introduced a shrinking projection method for solving split equilibrium problems and fixed point problems for asymptotically nonexpansive mappings in the framework of Hilbert spaces and proved some strong convergence theorems for the proposed new iterative method. They proved the following strong convergence theorem.

Theorem 1.1 (UAY). Let $\mathrm{H}_{1}, \mathrm{H}_{2}$ be two real Hilbert spaces and $\mathrm{C}, \mathrm{Q}$ be nonempty closed convex subsets of Hilbert spaces $\mathrm{H}_{1}$ and $\mathrm{H}_{2}$, respectively. Let $\mathrm{F}_{1}: \mathrm{C} \times \mathrm{C} \rightarrow \mathrm{R}$ and $\mathrm{F}_{2}: \mathrm{Q} \times \mathrm{Q} \rightarrow \mathrm{R}$ be two bifunctions satisfying conditions (A1)-(A4) and $\mathrm{F}_{2}$ be upper semi-continuous in the first argument. Let $\mathrm{T}: \mathrm{C} \rightarrow \mathrm{C}$ be an asymptotically nonexpansive mapping and $\mathrm{A}: \mathrm{H}_{1} \rightarrow \mathrm{H}_{2}$ be a bounded linear operator. Suppose that $\mathrm{F}(\mathrm{T}) \cap \Omega \neq \emptyset$, where $\Omega=\left\{v \in \mathrm{C}: v \in \mathrm{EP}\left(\mathrm{F}_{1}\right)\right.$ such that $\left.\mathrm{A} v \in \mathrm{EP}\left(\mathrm{F}_{2}\right)\right\}$ and let $\mathrm{x}_{0} \in \mathrm{C}$ define sequence $\left\{\mathrm{x}_{\mathrm{n}}\right\}$ iteratively as follows:

$$
\left\{\begin{array}{l}
C_{1}=C \\
u_{n}=T_{r_{n}}^{F_{1}}\left(I-\gamma A^{*}\left(I-T_{r_{n}}^{F_{2}}\right) A\right) x_{n} \\
y_{n}=\alpha_{n} x_{n}+\left(1-\alpha_{n}\right) T^{n} u_{n} \\
C_{n+1}=\left\{z \in C_{n}:\left\|y_{n}-z\right\|^{2} \leqslant\left\|x_{n}-z\right\|^{2}+\theta_{n}\right\} \\
x_{n+1}=P_{C_{n+1}} x_{0}
\end{array}\right.
$$

for each $\mathrm{n} \geqslant 1$, where $0 \leqslant \alpha_{n} \leqslant a<1,0<\mathrm{b} \leqslant \mathrm{r}_{\mathrm{n}}<\infty, \gamma \in\left(0, \frac{1}{\mathrm{~L}}\right)$ and

$$
\theta_{n}=\left(1-\alpha_{n}\right)\left(k_{n}^{2}-1\right) \sup \left\{\left\|x_{n}-z\right\|^{2}: z \in \Omega\right\},
$$

for all $n \geqslant 1, L$ is the spectral radius of the operator $A^{*} A$ and $A^{*}$ is the adjoint of $A$. Then the sequence $\left\{x_{n}\right\}$ converges strongly to a point $\mathrm{p} \in \mathrm{F}(\mathrm{T}) \cap \Omega$.

In 2015 [9], Guan et al. presented the following non-convex hybrid iteration algorithms and proved the following strong convergence theorem for a uniformly closed asymptotically family of countable quasi- $\mathrm{L}_{n}$-Lipschitz mappings.

Theorem 1.2 (G). Let $\mathrm{C}$ be a closed convex subset of a Hilbert space $\mathrm{H}$, and let $\left\{\mathrm{T}_{\mathrm{n}}\right\}: \mathrm{C} \rightarrow \mathrm{C}$ be a uniformly closed asymptotically family of countable quasi- $\mathrm{L}_{n}$-Lipschitz mappings from $\mathrm{C}$ into itself. Assume that $\alpha_{n} \in(\mathrm{a}, 1]$ holds for some $\mathrm{a} \in(0,1)$. Then $\left\{x_{\mathrm{n}}\right\}$ generated by

$$
\left\{\begin{array}{l}
x_{0} \in C=Q_{0}, \text { chosen arbitrarily, } \\
y_{n}=\left(1-\alpha_{n}\right) x_{n}+\alpha_{n} T_{n} x_{n}, \quad n \geqslant 0, \\
C_{n}=\left\{z \in C:\left\|y_{n}-z\right\| \leqslant\left(1+\left(L_{n}-1\right) \alpha_{n}\right)\left\|x_{n}-z\right\|\right\} \cap A, \quad n \geqslant 0, \\
Q_{n}=\left\{z \in Q_{n-1}:\left\langle x_{n}-z, x_{0}-x_{n}\right\rangle \geqslant 0\right\}, \quad n \geqslant 1, \\
x_{n+1}=P_{\overline{c o}} C_{n} \cap Q_{n} x_{0},
\end{array}\right.
$$

converges strongly to $\mathrm{P}_{\mathrm{F}} \mathrm{x}_{0}$, where $\overline{\mathrm{co}} \mathrm{C}_{\mathrm{n}}$ denotes the closed convex closure of $\mathrm{C}_{\mathrm{n}}$ for all $\mathrm{n} \geqslant 1, \mathrm{~A}=\{z \in \mathrm{H}$ : $\left.\left\|z-\mathrm{P}_{\mathrm{F}} \mathrm{x}_{0}\right\| \leqslant 1\right\}$.

The closely related works were also introduced in $[6,7,11,18]$.

In this paper, we introduce and consider a new multidirectional hybrid shrinking projection iterative algorithm (or called cloud hybrid shrinking projection iterative algorithm) for solving common problems which consist of generalized split equilibrium problems and fixed point problems for a family of countable quasi-Lipschitz mappings in the framework of Hilbert spaces. It is proved that under appropriate 
conditions, the sequence generated by the multidirectional hybrid shrinking projection method, converges strongly to some point which is the common fixed point of a family of countable quasi-Lipschitz mappings and the solution of the generalized split equilibrium problems. This iteration algorithm can accelerate the convergence speed of iterative sequence. The main results were also applied to solve split variational inequality problem and split optimization problems. Meanwhile, the main results were also used for solving common problems which consist of generalized split equilibrium problems and fixed point problems for asymptotically nonexpansive mappings. The results of this paper improve and extend the previous results given in the literature.

\section{Preliminaries}

In this section, we recall some concepts including the assumption which will be needed for the proof of our main result. Let $\mathrm{H}$ be a real Hilbert space with inner product $\langle\cdot, \cdot\rangle$ and norm $\|\cdot\|$. We write $x_{n} \rightarrow x$ to indicate that the sequence $\left\{x_{n}\right\}$ converges strongly to $x$. Let $C$ be a nonempty closed convex subset of $\mathrm{H}$, we denote by $\mathrm{P}_{\mathrm{C}}(\cdot)$ the metric projection onto $\mathrm{C}$. It is known that $z=\mathrm{P}_{\mathrm{C}}(\mathrm{x})$ is equivalent to that $z \in \mathrm{C}$ and $\langle z-y, x-z\rangle \geqslant 0$ for every $y \in C$. Recall that $T: C \rightarrow C$ is nonexpansive if $\|T x-T y\| \leqslant\|x-y\|$ for all $x, y \in C$. A point $x \in C$ is a fixed point of $T$ provided $T x=x$. Denote by $F(T)$ the set of fixed points of $T$, that is, $\mathrm{F}(\mathrm{T})=\{x \in \mathrm{C}: \mathrm{T} x=x\}$. It is will-known that $\mathrm{F}(\mathrm{T})$ is closed and convex. A mapping $\mathrm{T}: \mathrm{C} \rightarrow \mathrm{C}$ is said to be quasi-Lipschitz (or quasi-L-Lipschitz), if the following conditions hold:

(1) the fixed point set $F(T)$ is nonempty;

(2) $\|T x-p\| \leqslant L\|x-p\|$, for all $x \in C, p \in F(T)$,

where $1 \leqslant L<+\infty$ is a constant. $T$ is said to be quasi-nonexpansive, if $L=1$.

A sequence of mappings $\left\{T_{n}\right\}: C \rightarrow C$ is said to be a family of countable asymptotically quasi- $k_{n}{ }^{-}$ Lipschitz mappings, if the following hold:

(1) the common fixed point set $F$ is nonempty;

(2) $T_{n}$ is quasi- $k_{n}$-Lipschitz for each $n \geqslant 1$ and $\lim _{n \rightarrow \infty} k_{n}=1$.

Recall that a mapping $T: C \rightarrow C$ is said to be closed, if $x_{n} \rightarrow x$ and $\left\|T x_{n}-x_{n}\right\| \rightarrow 0$ as $n \rightarrow \infty$ implies $\mathrm{T} x=\mathrm{x}$.

Let $C$ be a nonempty closed and convex subset of a Hilbert space $H$. Let $\left\{T_{n}\right\}$ be sequence of mappings from $C$ into itself with a nonempty common fixed point set $F .\left\{T_{n}\right\}$ is said to be uniformly closed, if for any convergent sequence $\left\{z_{n}\right\} \subset C$ such that $\left\|T_{n} z_{n}-z_{n}\right\| \rightarrow 0$ as $n \rightarrow \infty$, the limit of $\left\{z_{n}\right\}$ belongs to $F$.

A mapping $\mathrm{T}: \mathrm{C} \times \mathrm{C} \rightarrow \mathrm{R}$ is said to be asymptotically nonexpansive, if there exists a sequence $\left\{k_{n}\right\} \subset[1, \infty)$ with $\lim _{n \rightarrow \infty} k_{n}=1$ such that

$$
\left\|T^{n} x-T^{n} y\right\| \leqslant k_{n}\|x-y\|,
$$

for all $x, y \in C$. It is easy to see that, if $k_{n} \equiv 1$, then $T$ is said to be nonexpansive. We denote the set of fixed point of $T$ by $F(T)$, that is, $F(T)=\{x \in C: T x=x\}$. There are many iterative methods for solving a fixed point problem corresponding to an asymptotically nonexpansive mapping (see also $[2,12,15,16]$ ).

Let $H$ be a Hilbert space and $C$ be a nonempty closed convex subset of $H$. For each $x \in H$, there exists a unique nearest point of $C$, denoted by $P_{C} x$, such that

$$
\left\|x-P_{C} x\right\| \leqslant\|x-y\|,
$$

for all $y \in C$. $P_{C} x$ is called the metric projection from $H$ onto $C$. It is well-known that $P_{C}$ is a firmly nonexpansive mapping from $\mathrm{H}$ onto $\mathrm{C}$, that is,

$$
\left\|P_{C} x-P_{C} y\right\|^{2} \leqslant\left\langle P_{C} x-P_{C} y, x-y\right\rangle,
$$


for all $x, y \in H$. Furthermore, for any $x \in H$ and $z \in C, z=P_{C} x$, if and only if

$$
\langle x-z, z-y\rangle \geqslant 0 \text {, }
$$

for all $y \in C$. A mapping $A: C \rightarrow H$ is called $\alpha$-inverse strongly monotone, if there exists $\alpha>0$ such that

$$
\langle x-y, A x-A y\rangle \geqslant \alpha\|A x-A y\|^{2},
$$

for all $x, y \in H$. Moreover, we can investigate that, for each $\lambda \in(0,2 \alpha], I-\lambda A$ is a nonexpansive mapping of $\mathrm{C}$ into $\mathrm{H}$ (see [11]).

Lemma 2.1. In a Hilbert space $\mathrm{H}$, the following identity holds:

$$
\|\lambda x+(1-\lambda) y\|^{2}=\lambda\|x\|^{2}+(1-\lambda)\|y\|^{2}-\lambda(1-\lambda)\|x-y\|^{2},
$$

for all $x, y \in \mathrm{H}$ and $\lambda \in[0,1]$.

Lemma 2.2 ([9]). Let $\mathrm{H}$ be a Hilbert space, let $\mathrm{C}$ be a closed convex subset of $\mathrm{E}$ and let $\left\{\mathrm{T}_{\mathrm{n}}\right\}$ be a uniformly closed asymptotically family of countable quasi- $\mathrm{k}_{\mathrm{n}}$-Lipschitz mappings from $\mathrm{C}$ into itself. Then the common fixed point set $\mathrm{F}$ is closed and convex.

Assumption 2.3 ([7]). Let $\mathrm{F}: \mathrm{C} \times \mathrm{C} \rightarrow \mathrm{R}$ be a bifunction satisfying the following conditions:

(A1) $F(x, y)=0$ for all $x \in C$;

(A2) $F$ is monotone, i.e., $F(x, y)+F(y, x) \leqslant 0$, for all $x, y \in C$;

(A3) for each $x, y, z \in C, \lim _{t \downarrow 0} F(t z+(1-t) x, y) \leqslant F(x, y)$;

(A4) for each $x \in C, y \longmapsto F(x, y)$ is convex and lower semi-continuous.

Lemma 2.4 ([7]). Let $\mathrm{C}$ be a nonempty closed convex subset of a Hilbert space $\mathrm{H}$ and $\mathrm{F}: \mathrm{C} \times \mathrm{C} \rightarrow \mathrm{R}$ be a bifunction which satisfies conditions (A1)-(A4). For any $\mathrm{x} \in \mathrm{H}$ and $\mathrm{r}>0$ define a mapping $\mathrm{T}_{\mathrm{r}}^{\mathrm{F}}: \mathrm{H} \rightarrow \mathrm{C}$ by

$$
\mathrm{T}_{\mathrm{r}}^{\mathrm{F}}(\mathrm{x})=\left\{z \in \mathrm{C}: \mathrm{F}(z, \mathrm{y})+\frac{1}{\mathrm{r}}\langle\mathrm{y}-z, z-x\rangle \geqslant 0, \forall y \in \mathrm{C}\right\} \text {. }
$$

Then $\mathrm{T}_{\mathrm{r}}^{\mathrm{F}}$ is well-defined and the following hold:

(1) $\mathrm{T}_{\mathrm{r}}^{\mathrm{F}}$ is single-valued;

(2) $\mathrm{T}_{\mathrm{r}}^{\mathrm{F}}$ is firmly nonexpansive, i.e., for any $\mathrm{x}, \mathrm{y} \in \mathrm{H}$,

$$
\left\|T_{r}^{F} x-T_{r}^{F} y\right\|^{2} \leqslant\left\langle T_{r}^{F} x-T_{r}^{F} y, x-y\right\rangle ;
$$

(3) $\mathrm{F}\left(\mathrm{T}_{\mathrm{r}}^{\mathrm{F}}\right)=\mathrm{EP}(\mathrm{F})$;

(4) $\mathrm{EP}(\mathrm{F})$ is closed and convex.

The following useful result is well-known.

Lemma 2.5. Let $\mathrm{H}$ be a Hilbert space, let $\mathrm{C}$ be a nonempty closed convex subset of $\mathrm{H}$ and let $\mathrm{x} \in \mathrm{E}$. Then

$$
\left\|z-\mathrm{P}_{\mathrm{C}} x\right\|^{2}+\left\|\mathrm{P}_{\mathrm{C}} x-x\right\|^{2} \leqslant\|z-x\|^{2}, \quad \forall z \in \mathrm{C} .
$$

\section{Main results}

Theorem 3.1. Let $\mathrm{H}_{1}, \mathrm{H}_{2}$ be two real Hilbert spaces and $\mathrm{C}, \mathrm{Q}$ be nonempty closed convex subsets of Hilbert spaces $\mathrm{H}_{1}$ and $\mathrm{H}_{2}$, respectively. Let $\mathrm{F}_{1}: \mathrm{C} \times \mathrm{C} \rightarrow \mathrm{R}$ and $\mathrm{F}_{2}: \mathrm{Q} \times \mathrm{Q} \rightarrow \mathrm{R}$ be two bifunctions satisfying conditions (A1)-(A4) and $\mathrm{F}_{2}$ be upper semi-continuous in the first argument. Let $\left\{\mathrm{T}_{\mathrm{n}}\right\}: \mathrm{C} \rightarrow \mathrm{C}$ be a uniformly closed and equicontinuous family of countable quasi- $\mathrm{k}_{\mathrm{n}}$-Lipschitz mappings with nonempty common fixed point set $\mathrm{F}$ and 
$A: \mathrm{H}_{1} \rightarrow \mathrm{H}_{2}$ be a bounded linear operator. Suppose that $\mathrm{F} \cap \Omega \neq \emptyset$, where $\Omega=\left\{v \in \mathrm{C}: v \in \mathrm{EP}\left(\mathrm{F}_{1}\right)\right.$ such that $\left.A v \in \operatorname{EP}\left(F_{2}\right)\right\}$ and let $x_{0, i} \in C, i=1,2,3, \cdots, N$, define sequences $\left\{x_{n, i}\right\}, i=1,2,3, \cdots, N$ and $\left\{\omega_{n}\right\}$ iteratively as follows:

$$
\left\{\begin{array}{l}
C_{1, i}=C, \quad i=1,2,3, \cdots, N, \\
u_{n, i}=T_{r_{n, i}}^{F_{1}}\left(I-\gamma_{i} A^{*}\left(I-T_{r_{n, i}}^{F_{2}}\right) A\right) x_{n, i}, \quad i=1,2,3, \cdots, N, \\
y_{n, i}=\alpha_{n, i} x_{n, i}+\left(1-\alpha_{n, i}\right) T_{n} u_{n, i}, \quad i=1,2,3, \cdots, N, \\
C_{n+1, i}=\left\{z \in C_{n, i}:\left\|y_{n, i}-z\right\|^{2} \leqslant\left\|x_{n, i}-z\right\|^{2}+\theta_{n, i}\right\}, \quad i=1,2,3, \cdots, N, \\
C_{n+1}=\bigcap_{i=1}^{N} C_{n+1, i} \\
x_{n+1, i}=P_{C_{n+1}} x_{0, i}, \quad i=1,2,3, \cdots, N, \\
\omega_{n+1}=\sum_{i=1}^{N} \lambda_{i} x_{n+1, i}, \quad \sum_{i=1}^{N} \lambda_{i}=1, \quad \lambda_{i} \in[0,1],
\end{array}\right.
$$

for each $n \geqslant 1$, where $0 \leqslant \alpha_{n, i} \leqslant a<1,0<b \leqslant r_{n, i}<\infty, \gamma_{i} \in\left(0, \frac{1}{L}\right)$ and

$$
\theta_{n, i}=\left(1-\alpha_{n, i}\right)\left(k_{n}^{2}-1\right) \sup \left\{\left\|x_{n, i}-z\right\|^{2}: z \in \Omega\right\},
$$

for all $\mathrm{n} \geqslant 1, i=1,2,3, \cdots, N, \mathrm{~L}$ is the spectral radius of the operator $\mathrm{A}^{*} \mathrm{~A}$ and $\mathrm{A}^{*}$ is the adjoint of $\mathrm{A}$. Then the following conclusions hold:

(1) $\left\{x_{n, i}\right\}$ converges strongly to $p_{i} \in F \cap \Omega$, for all $i=1,2,3, \cdots, N$;

(2) $\left\{\omega_{n}\right\}$ converges strongly to $\omega=\sum_{i=1}^{N} \lambda_{i} p_{i} \in F \cap \Omega$.

Proof. Firstly, we claim that, for each $n \geqslant 1, i=1,2,3, \cdots, N, A^{*}\left(I-T_{r_{n, i}}^{F_{2}}\right) A$ is a $\frac{1}{2 L}$-inverse strongly monotone mapping. Since $T_{r_{n, i}}^{F_{2}}$ is firmly nonexpansive and $\left(I-T_{r_{n, i}}^{F_{2}}\right.$ ) is $\frac{1}{2}$-inverse strongly monotone, it follows that

$$
\begin{aligned}
\left\|A^{*}\left(I-T_{r_{n, i}}^{F_{2}}\right) A x-A^{*}\left(I-T_{r_{n, i}}^{F_{2}}\right) A y\right\|^{2} & =\left\langle A^{*}\left(I-T_{r_{n, i}}^{F_{2}}\right)(A x-A y), A^{*}\left(I-T_{r_{n, i}}^{F_{2}}\right)(A x-A y)\right\rangle \\
& =\left\langle\left(I-T_{r_{n, i}}^{F_{2}}\right)(A x-A y), A A^{*}\left(I-T_{r_{n, i}}^{F_{2}}\right)(A x-A y)\right\rangle \\
& \leqslant L\left\langle\left(I-T_{r_{n, i}}^{F_{2}}\right)(A x-A y),\left(I-T_{r_{n, i}}^{F_{2}}\right)(A x-A y)\right\rangle \\
& =L\left\|\left(I-T_{r_{n, i}}^{F_{2}}\right)(A x-A y)\right\|^{2} \\
& \leqslant 2 L\left\langle x-y, A^{*}\left(I-T_{r_{n, i}}^{F_{2}}\right)(A x-A y)\right\rangle,
\end{aligned}
$$

for all $x, y \in H$, from which it can be concluded that

$$
\left\langle A^{*}\left(I-T_{r_{n, i}}^{F_{2}}\right) A x-A^{*}\left(I-T_{r_{n, i}}^{F_{2}}\right) A y, x-y\right\rangle \geqslant \frac{1}{2 L}\left\|A^{*}\left(I-T_{r_{n, i}}^{F_{2}}\right) A x-A^{*}\left(I-T_{r_{n, i}}^{F_{2}}\right) A y\right\|^{2},
$$

for all $x, y \in H$. That is, $A^{*}\left(I-T_{r_{n, i}}^{F_{2}}\right) A$ is a $\frac{1}{2 L}$-inverse strongly monotone mapping. Moreover, we claim that since $\gamma_{i} \in\left(0, \frac{1}{\mathrm{~L}}\right)$,

$$
I-\gamma_{i} A^{*}\left(I-T_{r_{n, i}}^{F_{2}}\right) A,
$$

are nonexpansive, for all $n \geqslant 1, i=1,2,3, \cdots, N$.

Next, we show that $F \cap \Omega \subset C_{n+1, i}$ for all $n \geqslant 1, i=1,2,3, \cdots, N$. Let $p \in F \cap \Omega$, i.e., $T_{r_{n, i}}^{F_{1}} p=p$ and $\left(I-\gamma_{i} A^{*}\left(I-T_{r_{n, i}}^{F_{2}}\right) A\right) p=p$. By mathematical induction, we have $p \in C=C_{1, i}$ and hence $F \cap \Omega \subset C_{1, i}$. Let $F \cap \Omega \subset C_{k, i}$, for some $k \geqslant 1$. It follows that

$$
\begin{aligned}
\left\|u_{k, i}-p\right\| & =\left\|T_{r_{k, i}}^{F_{1}}\left(I-\gamma_{i} A^{*}\left(I-T_{r_{k, i}}^{F_{2}}\right) A\right) x_{k, i}-T_{r_{k, i}}^{F_{1}}\left(I-\gamma_{i} A^{*}\left(I-T_{r_{k, i}}^{F_{2}}\right) A\right) p\right\| \\
& \leqslant\left\|\left(I-\gamma_{i} A^{*}\left(I-T_{r_{k, i}}^{F_{2}}\right) A\right) x_{k, i}-\left(I-\gamma_{i} A^{*}\left(I-T_{r_{k, i}}^{F_{2}}\right) A\right) p\right\| \\
& \leqslant\left\|x_{k, i}-p\right\|
\end{aligned}
$$


and

$$
\begin{aligned}
\left\|y_{k, i}-p\right\|^{2}= & \left\|\alpha_{k, i} x_{k}+\left(1-\alpha_{k, i}\right) T_{k} u_{k, i}-p\right\|^{2} \\
\leqslant & \alpha_{k, i}\left\|x_{k, i}-p\right\|^{2}+\left(1-\alpha_{k, i}\right)\left\|T_{k} u_{k, i}-p\right\|^{2} \\
& -\alpha_{k, i}\left(1-\alpha_{k, i}\right)\left\|x_{k, i}-p-\left(T_{k} u_{k, i}-T_{k} p\right)\right\|^{2} \\
\leqslant & \alpha_{k, i}\left\|x_{k, i}-p\right\|^{2}+\left(1-\alpha_{k, i}\right)\left\|u_{k, i}-p\right\|^{2} \\
& -\alpha_{k, i}\left(1-\alpha_{k, i}\right)\left\|x_{k, i}-T_{k} u_{k, i}\right\|^{2} \\
\leqslant & \alpha_{k, i}\left\|x_{k, i}-p\right\|^{2}+\left(1-\alpha_{k, i}\right) k_{k}^{2}\left\|x_{k, i}-p\right\|^{2} \\
= & \left\|x_{k}-p\right\|^{2}+\left(1-\alpha_{k, i}\right)\left(k_{k}^{2}-1\right)\left\|x_{k, i}-p\right\|^{2} \\
\leqslant & \left\|x_{k, i}-p\right\|^{2}+\left(1-\alpha_{k, i}\right)\left(k_{k}^{2}-1\right) M_{k, i}^{2} \\
= & \left\|x_{k, i}-p\right\|^{2}+\theta_{k, i}
\end{aligned}
$$

where $M_{k, i}=\sup \left\{\left\|x_{k, i}-z\right\|: z \in \Omega\right\}$ and $\theta_{k, i}=\left(1-\alpha_{k, i}\right)\left(k_{k}^{2}-1\right) M_{k, i}^{2}$. It can be concluded that $p \in C_{k+1, i}$ and $F(T) \cap \Omega \subset C_{k+1, i}$ and further, $F(T) \cap \Omega \subset C_{n+1, i}$ for all $n \geqslant 1, i=1,2,3, \cdots, N$. Therefore, $F(T) \cap \Omega \subset C_{n+1}=\cap_{i=1}^{N} C_{n+1, i}$, for all $n \geqslant 1$.

Next, we show that $C_{n}$ is closed and convex for all $n \geqslant 1$. It is obvious that, $C_{n, i}$ is closed for all $n \geqslant 1, i=1,2,3, \cdots, N$. Therefore, $C_{n}$ is closed for all $n \geqslant 1$. Suppose that $C_{k-1, i}$ is closed and convex for some $k \geqslant 2, i=1,2,3, \cdots, N$. We see for all $n \geqslant 1, i=1,2,3, \cdots, N$ that,

$$
\left\|y_{n, i}-z\right\|^{2} \leqslant\left\|x_{n, i}-z\right\|^{2}+\theta_{n, i}
$$

is equivalent to

$$
\left\langle z, 2\left(x_{n, i}-y_{n, i}\right)\right\rangle \leqslant\left\|x_{n, i}\right\|^{2}-\left\|y_{n, i}\right\|^{2}+\theta_{n, i} .
$$

Hence

$$
C_{k, i}=\left\{z \in C:\left\langle z, 2\left(x_{k, i}-y_{k, i}\right)\right\rangle \leqslant\left\|x_{k, i}\right\|^{2}-\left\|y_{k, i}\right\|^{2}+\theta_{k, i}\right\} \cap C_{k-1, i}
$$

is convex for all $i=1,2,3, \cdots, N$. By induction, we know that $C_{n, i}$ is convex for all $n \geqslant 1, i=$ $1,2,3, \cdots, N$. Therefore, $C_{n}$ is convex for all $n \geqslant 1$.

Next, from $x_{n, i}=P_{C_{n, i}} x_{0, i}, n \geqslant 1, i=1,2,3, \cdots, N$, we have

$$
\left\|x_{0}-x_{n, i}\right\| \leqslant\left\|x_{0}-y\right\|
$$

for all $y \in C_{n, i}$. Since $F(T) \cap \Omega$ is nonempty closed and convex, there exists $z_{0} \in F(T) \cap \Omega$, we have that

$$
\left\|x_{0}-x_{n, i}\right\| \leqslant\left\|x_{0}-z_{0}\right\| .
$$

This implies that $\left\{x_{n, i}\right\}$ is bounded. From $x_{n, i}=P_{C_{n, i}} x_{0, i}$ and $x_{n+1, i}=P_{C_{n+1, i}} x_{0, i} \in C_{n+1, i} \subset C_{n, i}$, we also have

$$
\left\|x_{n, i}-x_{0, i}\right\| \leqslant\left\|x_{n+1, i}-x_{0, i}\right\|,
$$

for all $n \in N$. This means that $\left\{\left\|x_{n, i}-x_{0}\right\|\right\}$ is bounded and nondecreasing. Then $\lim _{n \rightarrow \infty}\left\|x_{n, i}-x_{0}\right\|$ exists for all $i=1,2,3, \cdots, N$. Put $\lim _{n \rightarrow \infty}\left\|x_{n, i}-x_{0}\right\|=c_{i}$ for all $i=1,2,3, \cdots, N$. On the other hand, from $x_{n+m, i} \subset C_{n, i}$ for all $i=1,2,3, \cdots, N$, by using Lemma 2.5 , we have for any positive integer $m$, that

$$
\left\|x_{n+m, i}-x_{n, i}\right\|^{2} \leqslant\left\|x_{n+m, i}-x_{0, i}\right\|^{2}-\left\|x_{n, i}-x_{0, i}\right\|^{2} .
$$

So $\left\{x_{n, i}\right\}$ is a Cauchy sequence in $C$, then there exits a point $p_{i} \in C$ such that $\lim _{n \rightarrow \infty} x_{n, i}=p_{i}$ for any $i=1,2,3, \cdots, N$. Therefore,

$$
\omega_{n}=\sum_{i=1}^{N} \lambda_{i} x_{n, i} \rightarrow \omega=\sum_{i=1}^{N} \lambda_{i} p_{i},
$$

as $n \rightarrow \infty$. 
Since $\lim _{n \rightarrow \infty} x_{n, i}=p_{i}$ for any $i=1,2,3, \cdots, N$, we have

$$
\lim _{n \rightarrow \infty}\left\|x_{n, i}-x_{n+1, i}\right\|=0, \quad i=1,2,3, \cdots, N .
$$

Since $x_{n+1, i} \in C_{n+1, i} \subset C_{n, i}$ for any $i=1,2,3, \cdots, N$, we have

$$
\left\|y_{n, i}-x_{n+1, i}\right\|^{2} \leqslant\left\|x_{n, i}-x_{n+1, i}\right\|^{2}+\theta_{n, i}
$$

which means that

$$
\left\|y_{n, i}-x_{n+1, i}\right\| \leqslant\left\|x_{n, i}-x_{n+1, i}\right\|+\sqrt{\theta_{n, i}} .
$$

Thus, by (3.2) and (3.3) we have

$$
\left\|y_{n, i}-x_{n, i}\right\| \leqslant\left\|y_{n, i}-x_{n+1, i}\right\|+\left\|x_{n+1, i}-x_{n, i}\right\| \rightarrow 0,
$$

as $n \rightarrow \infty$. Furthermore, since $T_{r_{n, i}}^{F_{1}}$ is firmly nonexpansive, we have

$$
\begin{aligned}
\left\|u_{n, i}-p\right\|^{2}= & \left\|T_{r_{n, i}}^{F_{1}}\left(x_{n, i}-\gamma_{i} A^{*}\left(I-T_{r_{n, i}}^{F_{2}}\right) A x_{n, i}\right)-T_{r_{n, i}}^{F_{1}}\left(p-\gamma_{i} A^{*}\left(I-T_{r_{n, i}}^{F_{2}}\right) A p\right)\right\|^{2} \\
\leqslant & \left\|\left(I-\gamma_{i} A^{*}\left(I-T_{r_{n, i}}^{F_{2}}\right) A\right) x_{n, i}-\left(I-\gamma_{i} A^{*}\left(I-T_{r_{n, i}}^{F_{2}}\right) A\right) P\right\|^{2}-\|\left(I-T_{r_{n, i}}^{F_{1}}\right) \\
& \times\left(I-\gamma_{i} A^{*}\left(I-T_{r_{n, i}}^{F_{2}}\right) A\right) x_{n, i}-\left(I-T_{r_{n, i}}^{F_{1}}\right)-\left(I-\gamma_{i} A^{*}\left(I-T_{r_{n, i}}^{F_{2}}\right) A\right) p \|^{2} \\
= & \left\|x_{n, i}-p-\gamma_{i}\left(A^{*}\left(I-T_{r_{n, i}}^{F_{2}}\right) A x_{n, i}-A^{*}\left(I-T_{r_{n, i}}^{F_{2}}\right) A p\right)\right\|^{2}-\left\|\beta_{n, i}-T_{r_{n, i}}^{F_{1}} \beta_{n, i}\right\|^{2} \\
= & \left\|x_{n, i}-p\right\|^{2}-2 \gamma_{i}\left\langle x_{n, i}-p, A^{*}\left(I-T_{r_{n, i}}^{F_{2}}\right) A x_{n, i}-A^{*}\left(I-T_{r_{n, i}}^{F_{2}}\right) A p\right\rangle \\
& +\gamma_{i}^{2}\left\|A^{*}\left(I-T_{r_{n, i}}^{F_{2}}\right) A x_{n, i}-A^{*}\left(I-T_{r_{n, i}}^{F_{2}}\right) A p\right\|^{2}-\left\|\beta_{n, i}-T_{r_{n, i}}^{F_{1}} \beta_{n, i}\right\|^{2} \\
\leqslant & \left\|x_{n, i}-p\right\|^{2}+\gamma_{i}\left(\gamma_{i}-\frac{1}{L}\right)\left\|A^{*}\left(I-T_{r_{n, i}}^{F_{2}}\right) A x_{n, i}\right\|^{2}-\left\|\beta_{n, i}-T_{r_{n, i}}^{F_{1}} \beta_{n}\right\|^{2},
\end{aligned}
$$

where $\beta_{n, i}=\left(I-\gamma_{i} A^{*}\left(I-T_{r_{n, i}}^{F_{2}}\right) A\right) x_{n, i}$. Moreover,

$$
\begin{aligned}
\left\|y_{n, i}-p\right\|^{2}= & \left\|\alpha_{n, i} x_{n, i}+\left(1-\alpha_{n, i}\right) T_{n} u_{n, i}-p\right\|^{2} \\
\leqslant & \alpha_{n, i}\left\|x_{n, i}-p\right\|^{2}+\left(1-\alpha_{n, i}\right) k_{n}^{2}\left[\left\|x_{n, i}-p\right\|^{2}\right. \\
& \left.+\gamma_{i}\left(\gamma_{i}-\frac{1}{L}\right)\left\|A^{*}\left(I-T_{r_{n, i}}^{F_{2}}\right) A x_{n, i}\right\|^{2}-\left\|\beta_{n, i}-T_{r_{n, i}}^{F_{1}} \beta_{n, i}\right\|^{2}\right] \\
= & \alpha_{n, i}\left\|x_{n, i}-p\right\|^{2}+\left(1-\alpha_{n, i}\right) k_{n}^{2}\left\|x_{n, i}-p\right\|^{2}-\left(1-\alpha_{n, i}\right) k_{n}^{2}\left\|\beta_{n, i}-T_{r_{n, i}}^{F_{1}} \beta_{n, i}\right\|^{2} \\
& +\left(1-\alpha_{n, i}\right) k_{n}^{2} \gamma_{i}\left(\gamma_{i}-\frac{1}{L}\right)\left\|A^{*}\left(I-T_{r_{n, i}}^{F_{2}}\right) A x_{n, i}\right\|^{2},
\end{aligned}
$$

which leads to

$$
\begin{gathered}
\left(1-\alpha_{n, i}\right) k_{n}^{2}\left[\gamma_{i}\left(\frac{1}{L}-\gamma_{i}\right)\left\|A^{*}\left(I-T_{r_{n, i}}^{F_{2}}\right) A x_{n}\right\|^{2}+\left\|\beta_{n, i}-T_{r_{n, i}}^{F_{1}} \beta_{n, i}\right\|^{2}\right] \\
\leqslant\left(\alpha_{n, i}+\left(1-\alpha_{n, i}\right) k_{n}^{2}\right)\left\|x_{n, i}-p\right\|^{2}-\left\|y_{n, i}-p\right\|^{2} .
\end{gathered}
$$

Letting $\rho_{n}=k_{n}-1$, it is clear that $\rho_{n} \rightarrow 0$ as $n \rightarrow \infty$ and by (3.5), we have

$$
\begin{aligned}
\left(1-\alpha_{n, i}\right) k_{n}^{2} & {\left[\gamma_{i}\left(\frac{1}{L}-\gamma_{i}\right)\left\|A^{*}\left(I-T_{r_{n, i}}^{F_{2}}\right) A x_{n, i}\right\|^{2}+\left\|\beta_{n, i}-T_{r_{n, i}}^{F_{1}} \beta_{n, i}\right\|^{2}\right] } \\
& \leqslant \alpha_{n, i}\left\|x_{n, i}-p\right\|^{2}+\left(1-\alpha_{n, i}\right)\left(\rho_{n}+1\right)^{2}\left\|x_{n, i}-p\right\|^{2}-\left\|y_{n, i}-p\right\|^{2} \\
& \leqslant\left\|x_{n, i}-p\right\|^{2}-\left\|y_{n, i}-p\right\|^{2}+\left(1-\alpha_{n, i}\right)\left(\rho_{n}^{2}+2 \rho_{n}\right)\left\|x_{n, i}-p\right\|^{2} \\
& \leqslant\left(\left\|x_{n, i}-p\right\|+\left\|y_{n, i}-p\right\|\right)\left\|x_{n, i}-y_{n, i}\right\|+\left(1-\alpha_{n, i}\right)\left(\rho_{n}^{2}+2 \rho_{n}\right)\left\|x_{n, i}-p\right\|^{2} .
\end{aligned}
$$

By (3.4) and $\rho_{n} \rightarrow 0$ as $n \rightarrow \infty$, we have

$$
\left\|A^{*}\left(I-T_{r_{n, i}}^{F_{2}}\right) A x_{n, i}\right\|^{2} \rightarrow 0, \quad\left\|\beta_{n}-T_{r_{n, i}}^{F_{1}} \beta_{n, i}\right\|^{2} \rightarrow 0,
$$


as $n \rightarrow \infty$. Furthermore, since $A$ is linear bounded and so is $A^{*}$, we can conclude that

$$
\lim _{n \rightarrow \infty}\left\|\left(I-T_{r_{n, i}}^{F_{2}}\right) A x_{n, i}\right\|=0 .
$$

Next, we show that $\left\|u_{n, i}-x_{n, i}\right\| \rightarrow 0$. We investigate the following:

$$
\begin{aligned}
\left\|u_{n, i}-x_{n, i}\right\| & =\left\|T_{r_{n, i}}^{F_{1}} \beta_{n, i}-x_{n, i}\right\| \\
& \leqslant\left\|T_{r_{n, i}}^{F_{1}} \beta_{n, i}-\beta_{n, i}\right\|+\left\|\beta_{n, i}-x_{n, i}\right\| \\
& =\left\|T_{r_{n, i}}^{F_{1}} \beta_{n, i}-\beta_{n, i}\right\|+\left\|\left(I-\gamma_{i} A^{*}\left(I-T_{r_{n, i}}^{F_{2}}\right) A\right) x_{n, i}-x_{n, i}\right\| \\
& =\left\|T_{r_{n, i}}^{F_{1}} \beta_{n, i}-\beta_{n, i}\right\|+\gamma_{i}\left\|A^{*}\left(I-T_{r_{n, i}}^{F_{2}}\right) A x_{n, i}\right\| .
\end{aligned}
$$

Consequently, by (3.7), we can conclude that

$$
\left\|u_{n, i}-x_{n, i}\right\| \rightarrow 0 \text {. }
$$

Next, we show that $\left\|T_{n} x_{n, i}-x_{n, i}\right\| \rightarrow 0$. We consider

$$
\begin{aligned}
\left\|y_{n, i}-x_{n, i}\right\| & =\left\|\alpha_{n, i} x_{n, i}+\left(1-\alpha_{n, i}\right) T_{n} u_{n, i}-x_{n, i}\right\| \\
& =\left(1-\alpha_{n, i}\right)\left\|T_{n} u_{n, i}-x_{n, i}\right\|,
\end{aligned}
$$

and since

$$
\left\|y_{n, i}-x_{n+1, i}\right\| \leqslant\left\|x_{n, i}-x_{n+1, i}\right\|+\sqrt{\theta_{n, i}}
$$

hence,

$$
\begin{aligned}
\left\|T_{n} u_{n, i}-x_{n, i}\right\| & =\frac{1}{1-\alpha_{n, i}}\left\|y_{n, i}-x_{n, i}\right\| \\
& \leqslant \frac{1}{1-a}\left(\left\|y_{n, i}-x_{n+1, i}\right\|+\left\|x_{n+1, i}-x_{n, i}\right\|\right) \\
& \leqslant \frac{1}{1-a}\left(\left\|x_{n, i}-x_{n+1, i}\right\|+\sqrt{\theta_{n, i}}\right)+\frac{1}{1-a}\left\|x_{n+1, i}-x_{n, i}\right\|,
\end{aligned}
$$

and so $\left\|T_{n} u_{n, i}-x_{n, i}\right\| \rightarrow 0$. Consider

$$
\left\|T_{n} x_{n, i}-x_{n, i}\right\| \leqslant\left\|T_{n} x_{n, i}-T_{n} u_{n, i}\right\|+\left\|T_{n} u_{n, i}-x_{n, i}\right\| .
$$

Since $\left\{T_{n}\right\}$ is equicontinuous, we have

$$
\left\|T_{n} x_{n, i}-x_{n, i}\right\| \rightarrow 0,
$$

as $n \rightarrow \infty$. Since $\left\{T_{n}\right\}$ is uniformly closed, then $p_{i} \in F$ for all $i=1,2,3, \cdots, N$. Since $F$ is convex, we also have

$$
\omega=\lim _{n \rightarrow \infty} \omega_{n}=\lim _{n \rightarrow \infty} \sum_{i=1}^{N} \lambda_{i} x_{n, i}=\sum_{i=1}^{N} \lambda_{i} p_{i} \in F .
$$

Next, we show that $p_{i} \in \Omega$ for all $i=1,2,3, \cdots, N$. By (3.1),

$$
u_{n, i}=T_{r_{n, i}}^{F_{1}}\left(I-\gamma_{i} A^{*}\left(I-T_{r_{n, i}}^{F_{2}}\right) A\right) x_{n, i}
$$

that is,

$$
F_{1}\left(u_{n, i}, y\right)+\frac{1}{r_{n, i}}\left\langle y-u_{n, i}, u_{n, i}-x_{n, i}\right\rangle-\frac{1}{r_{n, i}}\left\langle y-u_{n, i}, \gamma_{i} A^{*}\left(T_{r_{n, i}}^{F_{2}}-I\right) A x_{n, i}\right\rangle \geqslant 0,
$$

for all $y \in C$. From (A2), it follows that

$$
-\frac{1}{r_{n, i}}\left\langle y-u_{n, i}, \gamma_{i} A^{*}\left(T_{r_{n, i}}^{F_{2}}-I\right) A x_{n, i}\right\rangle+\frac{1}{r_{n, i}}\left\langle y-u_{n, i}, u_{n, i}-x_{n, i}\right\rangle \geqslant F_{1}\left(y, u_{n, i}\right),
$$


for all $y \in C$. Since

$$
\left\|A^{*}\left(T_{r_{n, i}}^{F_{2}}-I\right) A x_{n, i}\right\| \rightarrow 0, \quad\left\|u_{n, i}-x_{n, i}\right\| \rightarrow 0, \quad\left\|x_{n, i}-p_{i}\right\| \rightarrow 0,
$$

as $n \rightarrow \infty$, we have

$$
F_{1}\left(y, p_{i}\right) \leqslant 0, \quad i=1,2,3, \cdots, N,
$$

for all $y \in C$. Let $y_{t}=t y+(1-t) p_{i}$, for any $0<t \leqslant 1$ and $y \in C$. It means that $y_{t} \in C$ and hence

$$
0=F_{1}\left(y_{t}, y_{t}\right) \leqslant t F_{1}\left(y_{t}, y\right)+(1-t) F_{1}\left(y, p_{i}\right) \leqslant t F_{1}\left(y_{t}, y\right),
$$

and then $F_{1}\left(y_{t}, y\right) \geqslant 0$. Letting $t \rightarrow 0$, we immediately have $F_{1}\left(p_{i}, y\right) \geqslant 0$, i.e.,

$$
p_{i} \in E P\left(F_{1}\right), \quad i=1,2,3, \cdots, N .
$$

Next, we show that $A p_{i} \in \operatorname{EP}\left(F_{2}\right)$ for all $i=1,2,3, \cdots, N$. Since $A$ is a bounded linear operator and (3.6), we have

$$
\left\|T_{r_{n, i}}^{F_{2}} A x_{n, i}-A p\right\| \leqslant\left\|T_{r_{n, i}}^{F_{2}} A x_{n, i}-A x_{n, i}\right\|+\left\|A x_{n, i}-A p\right\| \rightarrow 0,
$$

as $n \rightarrow \infty$, which yields that $T_{r_{n}}^{F_{2}} A x_{n} \rightarrow A p$. By the definition of $T_{r_{n}}^{F_{2}}$, we have

$$
F_{2}\left(T_{r_{n, i}}^{F_{2}} A x_{n, i}, y\right)+\frac{1}{r_{n, i}}\left\langle y-T_{r_{n, i}}^{F_{2}} A x_{n, i}, T_{r_{n, i}}^{F_{2}} A x_{n, i}-A x_{n, i}\right\rangle \geqslant 0
$$

for all $y \in C$. Since $F_{2}$ is upper semi-continuous in the first argument, taking limsup in (3.8), it follows that

$$
F_{2}\left(A p_{i}, y\right) \geqslant 0, \quad i=1,2,3, \cdots, N,
$$

for all $y \in C$, from which it can be concluded that

$$
A p_{i} \in \operatorname{EP}\left(F_{2}\right), \quad i=1,2,3, \cdots, N .
$$

So, $p_{i} \in \Omega$ for all $i=1,2,3, \cdots, N$. Consequently, since $\Omega$ is convex, we have $\omega=\sum_{i=1}^{N} \lambda_{i} p_{i} \in \Omega$. This completes the proof.

In Theorem 3.1, if the mapping $\mathrm{T}$ is a nonexpansive mapping, then we immediately have the following theorem.

Theorem 3.2. Let $\mathrm{H}_{1}, \mathrm{H}_{2}$ be two real Hilbert spaces and $\mathrm{C}, \mathrm{Q}$ be nonempty closed convex subsets of Hilbert spaces $\mathrm{H}_{1}$ and $\mathrm{H}_{2}$, respectively. Let $\mathrm{F}_{1}: \mathrm{C} \times \mathrm{C} \rightarrow \mathrm{R}$ and $\mathrm{F}_{2}: \mathrm{Q} \times \mathrm{Q} \rightarrow \mathrm{R}$ be two bifunctions satisfying conditions (A1)-(A4) and $\mathrm{F}_{2}$ be upper semi-continuous in the first argument. Let $\mathrm{T}: \mathrm{C} \rightarrow \mathrm{C}$ be a nonexpansive mapping and $A: H_{1} \rightarrow H_{2}$ be a bounded linear operator. Suppose that $\mathrm{F}(\mathrm{T}) \cap \Omega \neq \emptyset$, where $\Omega=\left\{v \in \mathrm{C}: v \in \mathrm{EP}\left(\mathrm{F}_{1}\right)\right.$ such that $\left.A v \in \operatorname{EP}\left(F_{2}\right)\right\}$ and let $x_{0, i} \in C, i=1,2,3, \cdots, N$ define sequences $\left\{x_{n, i}\right\}, i=1,2,3, \cdots, N$ and $\left\{\omega_{n}\right\}$ iteratively as follows:

$$
\left\{\begin{array}{l}
C_{1, i}=C, \quad i=1,2,3, \cdots, N, \\
u_{n, i}=T_{r_{n, i}}^{F_{1}}\left(I-\gamma_{i} A^{*}\left(I-T_{r_{n, i}}^{F_{2}}\right) A\right) x_{n, i}, \quad i=1,2,3, \cdots, N, \\
y_{n, i}=\alpha_{n, i} x_{n, i}+\left(1-\alpha_{n, i}\right) T u_{n, i}, \quad i=1,2,3, \cdots, N, \\
C_{n+1, i}=\left\{z \in C_{n, i}:\left\|y_{n, i}-z\right\| \leqslant\left\|x_{n, i}-z\right\|\right\}, \quad i=1,2,3, \cdots, N, \\
C_{n+1}=\bigcap_{i=1}^{N} C_{n+1, i} \\
x_{n+1, i}=P_{C_{n+1}} x_{0, i}, \quad i=1,2,3, \cdots, N, \\
\omega_{n+1}=\sum_{i=1}^{N} \lambda_{i} x_{n+1, i}, \quad \sum_{i=1}^{N} \lambda_{i}=1, \quad \lambda_{i} \in[0,1],
\end{array}\right.
$$

for each $n \geqslant 1$, where $0 \leqslant \alpha_{n, i} \leqslant a<1,0<b \leqslant r_{n, i}<\infty, \gamma_{i} \in\left(0, \frac{1}{L}\right)$ for all $n \geqslant 1, i=1,2,3, \cdots, N, L$ is the spectral radius of the operator $A^{*} A$ and $A^{*}$ is the adjoint of $A$. Then the following conclusions hold: 
(1) $\left\{x_{n, i}\right\}$ converges strongly to $p_{i} \in F(T) \cap \Omega$, for all $i=1,2,3, \cdots, N$;

(2) $\left\{\omega_{n}\right\}$ converges strongly to $\omega=\sum_{i=1}^{N} \lambda_{i} p_{i} \in F(T) \cap \Omega$.

Theorem 3.3. Let $\mathrm{H}_{1}, \mathrm{H}_{2}$ be two real Hilbert spaces and $\mathrm{C}, \mathrm{Q}$ be nonempty closed convex subsets of Hilbert spaces $\mathrm{H}_{1}$ and $\mathrm{H}_{2}$, respectively. Let $\mathrm{F}_{1}: \mathrm{C} \times \mathrm{C} \rightarrow \mathrm{R}$ and $\mathrm{F}_{2}: \mathrm{Q} \times \mathrm{Q} \rightarrow \mathrm{R}$ be two bifunctions satisfying conditions (A1)-(A4) and $\mathrm{F}_{2}$ be upper semi-continuous in the first argument. Let $\left\{\mathrm{T}_{\mathrm{n}}\right\}: \mathrm{C} \rightarrow \mathrm{C}$ be a uniformly closed and equicontinuous family of countable quasi- $\mathrm{k}_{\mathrm{n}}$-Lipschitz mappings with nonempty common fixed point set $\mathrm{F}$ and $A: \mathrm{H}_{1} \rightarrow \mathrm{H}_{2}$ be a bounded linear operator. Suppose that $\mathrm{F}(\mathrm{T}) \cap \Omega \neq \emptyset$, where $\Omega=\left\{v \in \mathrm{C}: v \in \mathrm{EP}\left(\mathrm{F}_{1}\right)\right.$ such that $\left.A v \in \operatorname{EP}\left(F_{2}\right)\right\}$ and let $x_{0, i} \in C, i=1,2,3, \cdots, N$, define sequences $\left\{x_{n, i}\right\}, i=1,2,3, \cdots, N$ and $\left\{\omega_{n}\right\}$ iteratively as follows:

$$
\left\{\begin{array}{l}
C_{1}=C \\
u_{n}=T_{r_{n}}^{F_{1}}\left(I-\gamma_{n} A^{*}\left(I-T_{r_{n}}^{F_{2}}\right) A\right) w_{n}, \\
y_{n}=\alpha_{n} w_{n}+\left(1-\alpha_{n}\right) T_{n} u_{n}, \\
C_{n+1}=\left\{z \in C_{n}:\left\|y_{n}-z\right\|^{2} \leqslant\left\|w_{n}-z\right\|^{2}+\theta_{n}\right\}, \\
x_{n, i}=P_{C_{n}} x_{0, i}, \quad i=1,2,3, \cdots, N, \\
\omega_{n}=\sum_{i=1}^{N} \lambda_{i} x_{n, i}, \quad \sum_{i=1}^{N} \lambda_{i}=1, \quad \lambda_{i} \in[0,1]
\end{array}\right.
$$

for each $n \geqslant 1$, where $0 \leqslant \alpha_{n} \leqslant a<1,0<b \leqslant r_{n}<\infty, \gamma_{n} \in\left(0, \frac{1}{L}\right)$ and

$$
\theta_{n}=\left(1-\alpha_{n}\right)\left(k_{n}^{2}-1\right) \sup \left\{\left\|w_{n}-z\right\|^{2}: z \in \Omega\right\},
$$

for all $n \geqslant 1, L$ is the spectral radius of the operator $A^{*} A$ and $A^{*}$ is the adjoint of $A$. Then the following conclusions hold:

(1) $\left\{x_{n, i}\right\}$ converges strongly to $p_{i} \in F \cap \Omega$ for all $i=1,2,3, \cdots, N$;

(2) $\left\{w_{n}\right\}$ converges strongly to $w=\sum_{i=1}^{N} \lambda_{i} p_{i} \in F \cap \Omega$.

Proof. Firstly, we claim that for each $n \geqslant 1, A^{*}\left(I-T_{r_{n}}^{F_{2}}\right) A$ is a $\frac{1}{2 L}$-inverse strongly monotone mapping. Since $T_{r_{n}}^{F_{2}}$ is firmly nonexpansive and $\left(I-T_{r_{n}}^{F_{2}}\right)$ is $\frac{1}{2}$-inverse strongly monotone, it follows that

$$
\begin{aligned}
\left\|A^{*}\left(I-T_{r_{n}}^{F_{2}}\right) A x-A^{*}\left(I-T_{r_{n}}^{F_{2}}\right) A y\right\|^{2} & =\left\langle A^{*}\left(I-T_{r_{n}}^{F_{2}}\right)(A x-A y), A^{*}\left(I-T_{r_{n}}^{F_{2}}\right)(A x-A y)\right\rangle \\
& =\left\langle\left(I-T_{r_{n}}^{F_{2}}\right)(A x-A y), A A^{*}\left(I-T_{r_{n}}^{F_{2}}\right)(A x-A y)\right\rangle \\
& \leqslant L\left\langle\left(I-T_{r_{n}}^{F_{2}}\right)(A x-A y),\left(I-T_{r_{n}}^{F_{2}}\right)(A x-A y)\right\rangle \\
& =L\left\|\left(I-T_{r_{n}}^{F_{2}}\right)(A x-A y)\right\|^{2} \\
& \leqslant 2 L\left\langle x-y, A^{*}\left(I-T_{r_{n}}^{F_{2}}\right)(A x-A y)\right\rangle,
\end{aligned}
$$

for all $x, y \in H$, from which it can be concluded that

$$
\left\langle A^{*}\left(I-T_{r_{n}}^{F_{2}}\right) A x-A^{*}\left(I-T_{r_{n}}^{F_{2}}\right) A y, x-y\right\rangle \geqslant \frac{1}{2 L}\left\|A^{*}\left(I-T_{r_{n}}^{F_{2}}\right) A x-A^{*}\left(I-T_{r_{n}}^{F_{2}}\right) A y\right\|^{2},
$$

for all $x, y \in H$. That is, $A^{*}\left(I-T_{r_{n}}^{F_{2}}\right) A$ is a $\frac{1}{2 L}$-inverse strongly monotone mapping. Moreover, we claim that since $\gamma_{n} \in\left(0, \frac{1}{L}\right)$,

$$
\mathrm{I}-\gamma_{n} A^{*}\left(\mathrm{I}-\mathrm{T}_{\mathrm{r}_{\mathrm{n}}}^{\mathrm{F}_{2}}\right) A
$$

are nonexpansive, for all $n \geqslant 1$.

Next, we show that $F(T) \cap \Omega \subset C_{n+1}$ for all $n \geqslant 1$. Let $p \in F(T) \cap \Omega$, i.e., $T_{r_{n}}^{F_{1}} p=p$ and $(I-$ $\left.\gamma_{n} A^{*}\left(I-T_{r_{n}}^{F_{2}}\right) A\right) p=p$. By mathematical induction, we have $p \in C=C_{1}$ and hence $F(T) \cap \Omega \subset C_{1}$. Let 
$\mathrm{F}(\mathrm{T}) \cap \Omega \subset \mathrm{C}_{\mathrm{k}}$ for some $\mathrm{k} \geqslant 1$. It follows that

and

$$
\begin{aligned}
\left\|u_{k}-p\right\| & =\left\|T_{r_{k}}^{F_{1}}\left(I-\gamma_{n} A^{*}\left(I-T_{r_{k}}^{F_{2}}\right) A\right) x_{k}-T_{r_{k}}^{F_{1}}\left(I-\gamma_{n} A^{*}\left(I-T_{r_{k}}^{F_{2}}\right) A\right) p\right\| \\
& \leqslant\left\|\left(I-\gamma_{n} A^{*}\left(I-T_{r_{k}}^{F_{2}}\right) A\right) x_{k}-\left(I-\gamma_{n} A^{*}\left(I-T_{r_{k}}^{F_{2}}\right) A\right) p\right\| \\
& \leqslant\left\|x_{k}-p\right\|,
\end{aligned}
$$

$$
\begin{aligned}
\left\|y_{k}-p\right\|^{2}= & \left\|\alpha_{k} w_{k}+\left(1-\alpha_{k}\right) T_{k} u_{k}-p\right\|^{2} \\
\leqslant & \alpha_{k}\left\|w_{k}-p\right\|^{2}+\left(1-\alpha_{k}\right)\left\|T_{k} u_{k}-p\right\|^{2} \\
& -\alpha_{k}\left(1-\alpha_{k}\right)\left\|x_{k}-p-\left(T^{k} u_{k}-T_{k} p\right)\right\|^{2} \\
\leqslant & \alpha_{k}\left\|w_{k}-p\right\|^{2}+\left(1-\alpha_{k}\right)\left\|u_{k}-p\right\|^{2} \\
& -\alpha_{k}\left(1-\alpha_{k}\right)\left\|w_{k}-T_{k} u_{k}\right\|^{2} \\
\leqslant & \alpha_{k}\left\|w_{k}-p\right\|^{2}+\left(1-\alpha_{k}\right) k_{k}^{2}\left\|x_{k}-p\right\|^{2} \\
= & \left\|x_{k}-p\right\|^{2}+\left(1-\alpha_{k}\right)\left(k_{k}^{2}-1\right)\left\|w_{k}-p\right\|^{2} \\
\leqslant & \left\|x_{k}-p\right\|^{2}+\left(1-\alpha_{k}\right)\left(k_{k}^{2}-1\right) M_{k}^{2} \\
= & \left\|w_{k}-p\right\|^{2}+\theta_{k},
\end{aligned}
$$

where $M_{k}=\sup \left\{\left\|w_{k}-z\right\|: z \in \Omega\right\}$ and $\theta_{k}=\left(1-\alpha_{k}\right)\left(k_{k}^{2}-1\right) M_{k}^{2}$. It can be concluded that $p \in C_{k+1}$ and $\mathrm{F} \cap \Omega \subset \mathrm{C}_{\mathrm{k}+1}$ and further, $\mathrm{F} \cap \Omega \subset \mathrm{C}_{\mathrm{n}+1}$, for all $\mathrm{n} \geqslant 1$.

Next, we show that $C_{n}$ is closed and convex for all $n \geqslant 1$. It is obvious that, $C_{n}$ is closed for all $n \geqslant 1$. Suppose that $C_{k-1}$ is closed and convex for some $k \geqslant 2$. We see for all $n \geqslant 1$ that,

$$
\left\|y_{n}-z\right\|^{2} \leqslant\left\|w_{n}-z\right\|^{2}+\theta_{n}
$$

is equivalent to

$$
\left\langle z, 2\left(w_{n}-y_{n}\right)\right\rangle \leqslant\left\|w_{n}\right\|^{2}-\left\|y_{n}\right\|^{2}+\theta_{n}
$$

Hence

$$
C_{k}=\left\{z \in C:\left\langle z, 2\left(w_{k}-y_{k}\right)\right\rangle \leqslant\left\|w_{k}\right\|^{2}-\left\|y_{k}\right\|^{2}+\theta_{k}\right\} \cap C_{k-1},
$$

is convex. By induction, we know that, $C_{n}$ is convex for all $n \geqslant 1$.

Next, from $x_{n, i}=P_{C_{n}} x_{0, i}, n \geqslant 1, i=1,2,3, \cdots, N$, we have

$$
\left\|x_{0}-x_{n, i}\right\| \leqslant\left\|x_{0}-y\right\|
$$

for all $y \in C_{n}$. Since $F(T) \cap \Omega$ is nonempty, closed and convex, there exists $z_{0} \in F(T) \cap \Omega$, we have that

$$
\left\|x_{0}-x_{n, i}\right\| \leqslant\left\|x_{0}-z_{0}\right\| .
$$

This implies that $\left\{x_{n, i}\right\}$ is bounded. From $x_{n, i}=P_{C_{n}} x_{0, i}$ and $x_{n+1, i}=P_{C_{n+1}} x_{0, i} \in C_{n+1} \subset C_{n}$, we also have

$$
\left\|x_{n, i}-x_{0, i}\right\| \leqslant\left\|x_{n+1, i}-x_{0, i}\right\|,
$$

for all $n \in N$, This means that $\left\{\left\|x_{n, i}-x_{0}\right\|\right\}$ is bounded and nondecreasing. Then $\lim _{n \rightarrow \infty}\left\|x_{n, i}-x_{0}\right\|$ exists for all $i=1,2,3, \cdots, N$. Put $\lim _{n \rightarrow \infty}\left\|x_{n, i}-x_{0}\right\|=c_{i}$ for all $i=1,2,3, \cdots, N$. On the other hand, from $x_{n+m, i} \subset C_{n}$ for all $i=1,2,3, \cdots, N$, by using Lemma 2.5 , we have for any positive integer $m$ that

$$
\left\|x_{n+m, i}-x_{n, i}\right\|^{2} \leqslant\left\|x_{n+m, i}-x_{0, i}\right\|^{2}-\left\|x_{n, i}-x_{0, i}\right\|^{2} .
$$


So $\left\{x_{n, i}\right\}$ is a Cauchy sequence in $C$, then there exits a point $p_{i} \in C$ such that $\lim _{n \rightarrow \infty} x_{n, i}=p_{i}$ for any $i=1,2,3, \cdots, N$. Therefore,

$$
w_{n}=\sum_{i=1}^{N} \lambda_{i} x_{n, i} \rightarrow w=\sum_{i=1}^{N} \lambda_{i} p_{i},
$$

as $n \rightarrow \infty$.

Since $\lim _{n \rightarrow \infty} w_{n}=w$, we have

$$
\lim _{n \rightarrow \infty}\left\|w_{n}-w_{n+1}\right\|=0
$$

Since $w_{n+1} \in C_{n+1} \subset C_{n}$, we have

$$
\left\|y_{n}-w_{n+1}\right\|^{2} \leqslant\left\|w_{n}-w_{n+1}\right\|^{2}+\theta_{n},
$$

which means that

$$
\left\|y_{n}-w_{n+1}\right\| \leqslant\left\|w_{n}-w_{n+1}\right\|+\sqrt{\theta_{n}} .
$$

Thus, by (3.10) and (3.11) we have

$$
\left\|y_{n}-w_{n}\right\| \leqslant\left\|y_{n}-w_{n+1}\right\|+\left\|w_{n+1}-x_{n}\right\| \rightarrow 0,
$$

as $n \rightarrow \infty$. Furthermore, since $T_{r_{n}}^{F_{1}}$ is firmly nonexpansive, we have

$$
\begin{aligned}
\left\|u_{n}-p\right\|^{2}= & \left\|T_{r_{n}}^{F_{1}}\left(w_{n}-\gamma_{n} A^{*}\left(I-T_{r_{n}}^{F_{2}}\right) A w_{n}\right)-T_{r_{n}}^{F_{1}}\left(p-\gamma_{n} A^{*}\left(I-T_{r_{n}}^{F_{2}}\right) A p\right)\right\|^{2} \\
\leqslant & \left\|\left(I-\gamma_{n} A^{*}\left(I-T_{r_{n}}^{F_{2}}\right) A\right) w_{n}-\left(I-\gamma_{n} A^{*}\left(I-T_{r_{n}}^{F_{2}}\right) A\right) P\right\|^{2}-\|\left(I-T_{r_{n}}^{F_{1}}\right) \\
& \times\left(I-\gamma_{n} A^{*}\left(I-T_{r_{n}}^{F_{2}}\right) A\right) w_{n}-\left(I-T_{r_{n}}^{F_{1}}\right)-\left(I-\gamma_{n} A^{*}\left(I-T_{r_{n}}^{F_{2}}\right) A\right) p \|^{2} \\
= & \left\|w_{n}-p-\gamma_{n}\left(A^{*}\left(I-T_{r_{n}}^{F_{2}}\right) A w_{n}-A^{*}\left(I-T_{r_{n}}^{F_{2}}\right) A p\right)\right\|^{2}-\left\|\beta_{n}-T_{r_{n}}^{F_{1}} \beta_{n}\right\|^{2} \\
= & \left\|w_{n}-p\right\|^{2}-2 \gamma_{n}\left\langle w_{n}-p, A^{*}\left(I-T_{r_{n}}^{F_{2}}\right) A w_{n}-A^{*}\left(I-T_{r_{n}}^{F_{2}}\right) A p\right\rangle \\
& +\gamma_{n}^{2}\left\|A^{*}\left(I-T_{r_{n}}^{F_{2}}\right) A w_{n}-A^{*}\left(I-T_{r_{n}}^{F_{2}}\right) A p\right\|^{2}-\left\|\beta_{n}-T_{r_{n}}^{F_{1}} \beta_{n}\right\|^{2} \\
\leqslant & \left\|w_{n}-p\right\|^{2}+\gamma_{n}\left(\gamma_{n}-\frac{1}{L}\right)\left\|A^{*}\left(I-T_{r_{n}}^{F_{2}}\right) A w_{n}\right\|^{2}-\left\|\beta_{n}-T_{r_{n}}^{F_{1}} \beta_{n}\right\|^{2},
\end{aligned}
$$

where $\beta_{n}=\left(I-\gamma_{n} A^{*}\left(I-T_{r_{n}}^{F_{2}}\right) A\right) w_{n}$. Moreover,

$$
\begin{aligned}
\left\|y_{n}-p\right\|^{2}= & \left\|\alpha_{n} w_{n}+\left(1-\alpha_{n}\right) T_{n} u_{n}-p\right\|^{2} \\
\leqslant & \alpha_{n}\left\|w_{n}-p\right\|^{2}+\left(1-\alpha_{n}\right) k_{n}^{2}\left[\left\|w_{n}-p\right\|^{2}\right. \\
& \left.+\gamma_{n}\left(\gamma_{n}-\frac{1}{L}\right)\left\|A^{*}\left(I-T_{r_{n}}^{F_{2}}\right) A w_{n}\right\|^{2}-\left\|\beta_{n}-T_{r_{n}}^{F_{1}} \beta_{n}\right\|^{2}\right] \\
= & \alpha_{n}\left\|w_{n}-p\right\|^{2}+\left(1-\alpha_{n}\right) k_{n}^{2}\left\|w_{n}-p\right\|^{2}-\left(1-\alpha_{n}\right) k_{n}^{2}\left\|\beta_{n}-T_{r_{n}}^{F_{1}} \beta_{n}\right\|^{2} \\
& +\left(1-\alpha_{n}\right) k_{n}^{2} \gamma_{n}\left(\gamma_{n}-\frac{1}{L}\right)\left\|A^{*}\left(I-T_{r_{n}}^{F_{2}}\right) A w_{n}\right\|^{2},
\end{aligned}
$$

which leads to

$$
\begin{aligned}
& \left(1-\alpha_{n}\right) k_{n}^{2}\left[\gamma_{n}\left(\frac{1}{L}-\gamma_{n}\right)\left\|A^{*}\left(I-T_{r_{n}}^{F_{2}}\right) A w_{n}\right\|^{2}+\left\|\beta_{n}-T_{r_{n}}^{F_{1}} \beta_{n}\right\|^{2}\right] \\
& \leqslant\left(\alpha_{n}+\left(1-\alpha_{n}\right) k_{n}^{2}\right)\left\|w_{n}-p\right\|^{2}-\left\|y_{n}-p\right\|^{2} .
\end{aligned}
$$

Letting $\rho_{n}=k_{n}-1$, it is clear that $\rho_{n} \rightarrow 0$ as $n \rightarrow \infty$ and, by (3.13), we have

$$
\begin{aligned}
\left(1-\alpha_{n}\right) k_{n}^{2} & {\left[\gamma_{n}\left(\frac{1}{L}-\gamma_{n}\right)\left\|A^{*}\left(I-T_{r_{n}}^{F_{2}}\right) A w_{n}\right\|^{2}+\left\|\beta_{n}-T_{r_{n}}^{F_{1}} \beta_{n}\right\|^{2}\right] } \\
& \leqslant \alpha_{n}\left\|w_{n}-p\right\|^{2}+\left(1-\alpha_{n}\right)\left(\rho_{n}+1\right)^{2}\left\|w_{n}-p\right\|^{2}-\left\|y_{n}-p\right\|^{2} \\
& \leqslant\left\|w_{n}-p\right\|^{2}-\left\|y_{n}-p\right\|^{2}+\left(1-\alpha_{n}\right)\left(\rho_{n}^{2}+2 \rho_{n}\right)\left\|w_{n}-p\right\|^{2} \\
& \leqslant\left(\left\|w_{n}-p\right\|+\left\|y_{n}-p\right\|\right)\left\|w_{n}-y_{n}\right\|+\left(1-\alpha_{n}\right)\left(\rho_{n}^{2}+2 \rho_{n}\right)\left\|w_{n}-p\right\|^{2} .
\end{aligned}
$$


By (3.12) and $\rho_{n} \rightarrow 0$ as $n \rightarrow \infty$, we have

$$
\left\|A^{*}\left(\mathrm{I}-\mathrm{T}_{\mathrm{r}_{\mathrm{n}}}^{\mathrm{F}_{2}}\right) A w_{\mathrm{n}}\right\|^{2} \rightarrow 0, \quad\left\|\beta_{\mathrm{n}}-\mathrm{T}_{\mathrm{r}_{\mathrm{n}}}^{\mathrm{F}_{1}} \beta_{\mathrm{n}}\right\|^{2} \rightarrow 0,
$$

as $n \rightarrow \infty$. Furthermore, since $A$ is linear bounded and so is $A^{*}$, we can conclude that

$$
\lim _{n \rightarrow \infty}\left\|\left(I-T_{r_{n}}^{F_{2}}\right) A w_{n}\right\|=0 .
$$

Next, we show that $\left\|u_{n}-w_{n}\right\| \rightarrow 0$. We investigate the following:

$$
\begin{aligned}
\left\|u_{n}-w_{n}\right\| & =\left\|T_{r_{n}}^{F_{1}} \beta_{n}-w_{n}\right\| \\
& \leqslant\left\|T_{r_{n}}^{F_{1}} \beta_{n}-\beta_{n}\right\|+\left\|\beta_{n}-w_{n}\right\| \\
& =\left\|T_{r_{n}}^{F_{1}} \beta_{n}-\beta_{n}\right\|+\left\|\left(I-\gamma_{n} A^{*}\left(I-T_{r_{n}}^{F_{2}}\right) A\right) w_{n}-w_{n}\right\| \\
& =\left\|T_{r_{n}}^{F_{1}} \beta_{n}-\beta_{n}\right\|+\gamma_{n}\left\|A^{*}\left(I-T_{r_{n}}^{F_{2}}\right) A w_{n}\right\| .
\end{aligned}
$$

Consequently, by (3.15), we can conclude that

$$
\left\|u_{n}-w_{n}\right\| \rightarrow 0
$$

Next, we show that $\left\|T_{n} w_{n}-w_{n}\right\| \rightarrow 0$. We consider

$$
\begin{aligned}
\left\|y_{n}-w_{n}\right\| & =\left\|\alpha_{n} w_{n}+\left(1-\alpha_{n}\right) T_{n} u_{n}-w_{n}\right\| \\
& =\left(1-\alpha_{n}\right)\left\|T_{n} u_{n}-w_{n}\right\|,
\end{aligned}
$$

and since

$$
\left\|y_{n}-w_{n+1}\right\| \leqslant\left\|w_{n}-w_{n+1}\right\|+\sqrt{\theta_{n}} .
$$

Hence,

$$
\begin{aligned}
\left\|T_{n} u_{n}-w_{n}\right\| & =\frac{1}{1-\alpha_{n}}\left\|y_{n}-w_{n}\right\| \\
& \leqslant \frac{1}{1-a}\left(\left\|y_{n}-w_{n+1}\right\|+\left\|w_{n+1}-w_{n}\right\|\right) \\
& \leqslant \frac{1}{1-a}\left(\left\|w_{n}-w_{n+1}\right\|+\sqrt{\theta_{n}}\right)+\frac{1}{1-a}\left\|w_{n+1}-w_{n}\right\|,
\end{aligned}
$$

and so $\left\|T_{n} u_{n}-w_{n}\right\| \rightarrow 0$. Consider

$$
\left\|\mathrm{T}_{n} w_{n}-w_{n}\right\| \leqslant\left\|\mathrm{T}_{n} w_{n}-\mathrm{T}_{n} u_{n}\right\|+\left\|\mathrm{T}_{n} u_{n}-w_{n}\right\| .
$$

Since $\left\{T_{n}\right\}$ is equicontinuous, we have

$$
\left\|\mathrm{T}_{\mathrm{n}} w_{\mathrm{n}}-w_{\mathrm{n}}\right\| \rightarrow 0,
$$

as $n \rightarrow \infty$. Since $\left\{T_{n}\right\}$ is uniformly closed, then $w \in F$.

Next, we show that $w \in \Omega$ for all $i=1,2,3, \cdots, N$. By (3.9),

$$
u_{n}=T_{r_{n}}^{F_{1}}\left(I-\gamma_{n} A^{*}\left(I-T_{r_{n}}^{F_{2}}\right) A\right) w_{n},
$$

that is,

$$
F_{1}\left(u_{n}, y\right)+\frac{1}{r_{n}}\left\langle y-u_{n}, u_{n}-w_{n}\right\rangle-\frac{1}{r_{n}}\left\langle y-u_{n}, \gamma_{n} A^{*}\left(T_{r_{n}}^{F_{2}}-I\right) A w_{n}\right\rangle \geqslant 0,
$$

for all $y \in C$. From (A2), it follows that

$$
-\frac{1}{r_{n}}\left\langle y-u_{n}, \gamma_{n} A^{*}\left(T_{r_{n}}^{F_{2}}-I\right) A w_{n}\right\rangle+\frac{1}{r_{n}}\left\langle y-u_{n}, u_{n}-w_{n}\right\rangle \geqslant F_{1}\left(y, u_{n}\right),
$$


for all $y \in C$. Since

$$
\left\|A^{*}\left(\mathrm{~T}_{r_{n}}^{\mathrm{F}_{2}}-\mathrm{I}\right) A w_{\mathrm{n}}\right\| \rightarrow 0, \quad\left\|\mathrm{u}_{\mathrm{n}}-w_{\mathrm{n}}\right\| \rightarrow 0, \quad\left\|w_{\mathrm{n}}-w\right\| \rightarrow 0,
$$

as $n \rightarrow \infty$, we have

$$
F_{1}(y, w) \leqslant 0,
$$

for all $y \in C$. Let $y_{t}=t y+(1-t) p_{i}$ for any $0<t \leqslant 1$ and $y \in C$. It means that $y_{t} \in C$ and hence

$$
0=F_{1}\left(y_{t}, y_{t}\right) \leqslant t F_{1}\left(y_{t}, y\right)+(1-t) F_{1}(y, w) \leqslant t F_{1}\left(y_{t}, y\right)
$$

and then $F_{1}\left(y_{t}, y\right) \geqslant 0$. Letting $t \rightarrow 0$, we immediately have $F_{1}(w, y) \geqslant 0$, i.e.,

$$
w \in \operatorname{EP}\left(F_{1}\right)
$$

Next, we show that $A w \in \operatorname{EP}\left(F_{2}\right)$. Since $A$ is a bounded linear operator and (3.14), we have

$$
\left\|T_{r_{n}}^{F_{2}} A w_{n}-A p\right\| \leqslant\left\|T_{r_{n}}^{F_{2}} A w_{n}-A w_{n}\right\|+\left\|A w_{n}-A p\right\| \rightarrow 0
$$

as $n \rightarrow \infty$, which yields that $T_{r_{n}}^{F_{2}} A w_{n} \rightarrow A w$. By the definition of $T_{r_{n}}^{F_{2}}$, we have

$$
F_{2}\left(T_{r_{n}}^{F_{2}} A w_{n}, y\right)+\frac{1}{r_{n}}\left\langle y-T_{r_{n}}^{F_{2}} A w_{n}, T_{r_{n}}^{F_{2}} A w_{n}-A w_{n}\right\rangle \geqslant 0
$$

for all $y \in C$. Since $F_{2}$ is upper semi-continuous in the first argument, taking limsup in (3.16), it follows that

$$
F_{2}(A w, y) \geqslant 0,
$$

for all $y \in C$, from which it can be concluded that

$$
A w \in \operatorname{EP}\left(F_{2}\right) .
$$

So, $w \in \Omega$. This completes the proof.

Remark 3.4. Let $\mathrm{T}: \mathrm{C} \rightarrow \mathrm{C}$ be an asymptotically nonexpansive mapping and let $\mathrm{T}_{\mathrm{n}}=\mathrm{T}^{\mathrm{n}}$ for all $\mathrm{n} \geqslant 1$. It is easy to check that the $\left\{T_{n}\right\}$ is a uniformly closed and equicontinuous family of countable quasi- $k_{n}-$ Lipschitz mappings with nonempty common fixed point set $F=F(T)$. Where $k_{n} \rightarrow 1$ and

$$
\left\|T^{n} x-T^{n} y\right\| \leqslant k_{n}\|x-y\|, \quad \forall x, y \in C .
$$

Therefore, Theorem 3.1 and Theorem 3.3 still hold for $\left\{T_{n}\right\}$ is replaced by $\left\{T^{n}\right\}$, where $T$ is an asymptotically nonexpansive mapping.

\section{Applications to split variational inequality problems}

Firstly, we point out the so-called variational inequality problem (shortly, VIP), which is to find a point $x^{*} \in \mathrm{C}$ which satisfies the following inequality:

$$
\left\langle A x^{*}, z-x^{*}\right\rangle \geqslant 0,
$$

for all $z \in \mathrm{C}$. Its solution set is symbolized by $\mathrm{VI}(\mathrm{A}, \mathrm{C})$.

In 2012, Censor et al. [4] proposed the split variational inequality problem (shortly, SVIP) which is formulated as follows:

Find a point $x^{*} \in \mathrm{C}$ such that $\left\langle f\left(x^{*}\right), x-x^{*}\right\rangle \geqslant 0$, for all $x \in C$, 
and such that

$$
\mathrm{y}^{*}=A x^{*} \in Q \text { solves }\left\langle g\left(y^{*}\right), y-y^{*}\right\rangle \geqslant 0, \quad \text { for all } y \in Q \text {, }
$$

where $A: C \rightarrow C$ is a bounded linear operator. The solution set of split variational inequality problem is denoted by the SVIP.

Setting $F_{1}(x, y)=\langle f(x), y-x\rangle$ and $F_{2}(x, y)=\langle g(x), y-x\rangle$, it is clear that $F_{1}, F_{2}$ satisfy conditions (A1)-(A4), where $f$ and $g$ are $\eta_{1}$ - and $\eta_{2}$-inverse strongly monotone mappings, respectively.

From Lemma 2.4, we define a mapping $\mathrm{T}_{\mathrm{r}}^{\mathrm{f}}: \mathrm{H} \rightarrow \mathrm{C}$ by

$$
\mathrm{T}_{\mathrm{r}}^{\mathrm{f}}(\mathrm{x})=\left\{z \in \mathrm{C}:\langle\mathrm{f}(\mathrm{x}), \mathrm{y}-\mathrm{x}\rangle+\frac{1}{\mathrm{r}}\langle\mathrm{y}-z, z-x\rangle \geqslant 0, \forall \mathrm{y} \in \mathrm{C}\right\},
$$

and $\mathrm{T}_{\mathrm{r}}^{\mathrm{g}}: \mathrm{H} \rightarrow \mathrm{C}$ by

$$
\mathrm{T}_{\mathrm{r}}^{\mathrm{g}}(\mathrm{x})=\left\{z \in \mathrm{C}:\langle\mathrm{g}(\mathrm{x}), \mathrm{y}-\mathrm{x}\rangle+\frac{1}{\mathrm{r}}\langle\mathrm{y}-z, z-x\rangle \geqslant 0, \forall \mathrm{y} \in \mathrm{C}\right\} .
$$

Then, by Theorem 3.1, we get the following theorem.

Theorem 4.1. Let $\mathrm{H}_{1}, \mathrm{H}_{2}$ be two real Hilbert spaces and $\mathrm{C}, \mathrm{Q}$ be nonempty closed convex subsets of Hilbert spaces $\mathrm{H}_{1}$ and $\mathrm{H}_{2}$, respectively. Let $\mathrm{f}$ and $\mathrm{g}$ be $\eta_{1}$ - and $\eta_{2}$-inverse strongly monotone mappings, respectively. Let $\left\{\mathrm{T}_{\mathrm{n}}\right\}$ be a uniformly closed and equicontinuous family of countable quasi- $\mathrm{k}_{\mathrm{n}}$-Lipschitz mappings with nonempty common fixed point set $\mathrm{F}$ and $\mathrm{A}: \mathrm{H}_{1} \rightarrow \mathrm{H}_{2}$ be a bounded linear operator. Suppose that $\mathrm{F}(\mathrm{T}) \cap \Omega \neq \emptyset$, where $\Omega=\{v \in C: v \in \operatorname{SVIP}(f)$ such that $A v \in \operatorname{SVIP}(g)\}$ and let $x_{0, i} \in \mathrm{C}, \mathrm{i}=1,2,3, \cdots, \mathrm{N}$, define sequences $\left\{x_{n, i}\right\}, i=1,2,3, \cdots, N$ and $\left\{\omega_{n}\right\}$ iteratively as follows:

$$
\left\{\begin{array}{l}
C_{1, i}=C, \quad i=1,2,3, \cdots, N, \\
u_{n, i}=T_{r_{n, i}}^{f}\left(I-\gamma_{i} A^{*}\left(I-T_{r_{n, i}}^{g}\right) A\right) x_{n, i}, \quad i=1,2,3, \cdots, N, \\
y_{n, i}=\alpha_{n, i} x_{n, i}+\left(1-\alpha_{n, i}\right) T_{n} u_{n, i}, \quad i=1,2,3, \cdots, N, \\
C_{n+1, i}=\left\{z \in C_{n, i}:\left\|y_{n, i}-z\right\|^{2} \leqslant\left\|x_{n, i}-z\right\|^{2}+\theta_{n, i}\right\}, \quad i=1,2,3, \cdots, N, \\
C_{n+1}=\bigcap_{i=1}^{N} C_{n+1, i} \\
x_{n+1, i}=P_{C_{n+1}} x_{0, i}, \quad i=1,2,3, \cdots, N, \\
\omega_{n+1}=\sum_{i=1}^{N} \lambda_{i} x_{n+1, i}, \quad \sum_{i=1}^{N} \lambda_{i}=1, \quad \lambda_{i} \in[0,1]
\end{array}\right.
$$

for each $n \geqslant 1$, where $0 \leqslant \alpha_{n, i} \leqslant a<1,0<b \leqslant r_{n, i}<\infty, \gamma_{i} \in\left(0, \frac{1}{L}\right)$ and

$$
\theta_{n, i}=\left(1-\alpha_{n, i}\right)\left(k_{n}^{2}-1\right) \sup \left\{\left\|x_{n, i}-z\right\|^{2}: z \in \Omega\right\},
$$

for all $\mathrm{n} \geqslant 1, i=1,2,3, \cdots, N, L$ is the spectral radius of the operator $A^{*} A$ and $A^{*}$ is the adjoint of $A$. Then the following conclusions hold:

(1) $\left\{x_{n, i}\right\}$ converges strongly to $p_{i} \in F \cap \Omega$ for all $i=1,2,3, \cdots, N$;

(2) $\left\{\omega_{n}\right\}$ converges strongly to $\omega=\sum_{i=1}^{N} \lambda_{i} p_{i} \in F \cap \Omega$.

Proof. The desired result can be proved directly through Theorem 3.1.

Then, by Theorem 3.3, we get the following result.

Theorem 4.2. Let $\mathrm{H}_{1}, \mathrm{H}_{2}$ be two real Hilbert spaces and $\mathrm{C}, \mathrm{Q}$ be nonempty closed convex subsets of Hilbert spaces $\mathrm{H}_{1}$ and $\mathrm{H}_{2}$, respectively. Let $\mathrm{f}$ and $\mathrm{g}$ be $\eta_{1}$ - and $\eta_{2}$-inverse strongly monotone mappings, respectively. Let $\left\{\mathrm{T}_{\mathrm{n}}\right\}$ be a uniformly closed and equicontinuous family of countable quasi- $\mathrm{k}_{\mathrm{n}}$-Lipschitz mappings with nonempty common fixed point set $\mathrm{F}$ and $\mathrm{A}: \mathrm{H}_{1} \rightarrow \mathrm{H}_{2}$ be a bounded linear operator. Suppose that $\mathrm{F}(\mathrm{T}) \cap \Omega \neq \emptyset$, where 
$\Omega=\{v \in \mathrm{C}: v \in \operatorname{SVIP}(\mathrm{f})$ such that $\mathrm{A} v \in \operatorname{SVIP}(\mathrm{g})\}$ and let $x_{0, i} \in \mathrm{C}, \mathrm{i}=1,2,3, \cdots, \mathrm{N}$, define sequences $\left\{x_{n, i}\right\}, i=1,2,3, \cdots, N$, and $\left\{\omega_{n}\right\}$ iteratively as follows:

$$
\left\{\begin{array}{l}
C_{1}=C \\
u_{n}=T_{r_{n}}^{f}\left(I-\gamma_{n} A^{*}\left(I-T_{r_{n}}^{g}\right) A\right) w_{n} \\
y_{n}=\alpha_{n} w_{n}+\left(1-\alpha_{n}\right) T_{n} u_{n} \\
C_{n+1}=\left\{z \in C_{n}:\left\|y_{n}-z\right\|^{2} \leqslant\left\|w_{n}-z\right\|^{2}+\theta_{n}\right\} \\
x_{n, i}=P_{C_{n}} x_{0, i}, \quad i=1,2,3, \cdots, N, \\
\omega_{n}=\sum_{i=1}^{N} \lambda_{i} x_{n, i}, \quad \sum_{i=1}^{N} \lambda_{i}=1, \quad \lambda_{i} \in[0,1]
\end{array}\right.
$$

for each $\mathrm{n} \geqslant 1$, where $0 \leqslant \alpha_{\mathrm{n}} \leqslant \mathrm{a}<1,0<\mathrm{b} \leqslant \mathrm{r}_{\mathrm{n}}<\infty, \gamma_{\mathrm{n}} \in\left(0, \frac{1}{\mathrm{~L}}\right)$ and

$$
\theta_{\mathrm{n}}=\left(1-\alpha_{\mathrm{n}}\right)\left(k_{\mathrm{n}}^{2}-1\right) \sup \left\{\left\|w_{\mathrm{n}}-z\right\|^{2}: z \in \Omega\right\},
$$

for all $n \geqslant 1, L$ is the spectral radius of the operator $A^{*} A$ and $A^{*}$ is the adjoint of $A$. Then the following conclusions hold:

(1) $\left\{x_{n, i}\right\}$ converges strongly to $p_{i} \in \mathrm{F} \cap \Omega$ for all $i=1,2,3, \cdots, N$;

(2) $\left\{\omega_{n}\right\}$ converges strongly to $\omega=\sum_{i=1}^{N} \lambda_{i} p_{i} \in F \cap \Omega$.

Proof. The desired result can be proved directly through Theorem 3.3.

\section{Applications to split optimization problems}

In this section, we mention applications to the split optimization problem, which is to find $x^{*} \in \mathrm{C}$ such that

$$
f\left(x^{*}\right) \geqslant f(x) \text { for all } x \in C \text { satisfying } A x^{*}=y^{*} \in Q \text { solves } g\left(y^{*}\right) \geqslant g(y),
$$

for all $y \in Q$. We symbolize $O(f, A)$ for the solution set of the split optimization problem.

Let $f: C \rightarrow R$ and $g: Q \rightarrow R$ be two functions satisfying the following assumption:

(1) $f(t x+(1-t) y) \leqslant f(y), g(t u+(1-t) v) \leqslant g(v), \forall x, y \in C, u, v \in Q$

(2) $f(x), g(u)$ are concave and upper semi-continuous.

Let $F_{1}(x, y)=f(x)-f(y)$ for all $x, y \in C$ and $F_{2}(u, v)=g(u)-g(v)$ for all $u, v \in Q$. If $f$ and $g$ satisfy conditions (1) and (2), then it is clear that $F_{1}: C \times C \rightarrow R$ and $F_{2}: Q \times Q \rightarrow R$ are two bifunctions satisfying conditions (A1)-(A4). Let $\mathrm{T}$ be an asymptotically nonexpansive mapping defined on a bounded closed convex subset $\mathrm{C}$ of a Hilbert space $\mathrm{H}$. By Lemma 2.4, we define a mapping $\mathrm{O}_{\mathrm{r}}^{\mathrm{f}}: \mathrm{H} \rightarrow \mathrm{C}$ by

$$
\mathrm{O}_{\mathrm{r}}^{\mathrm{f}}(x)=\left\{z \in \mathrm{C}: \mathrm{f}(\mathrm{x})-\mathrm{f}(\mathrm{y})+\frac{1}{\mathrm{r}}\langle\mathrm{y}-z, z-x\rangle \geqslant 0, \forall \mathrm{y} \in \mathrm{C}\right\},
$$

and $\mathrm{O}_{\mathrm{r}}^{\mathrm{g}}: \mathrm{H} \rightarrow \mathrm{C}$ by

$$
\mathrm{O}_{\mathrm{r}}^{\mathrm{g}}(x)=\left\{z \in \mathrm{C}: \mathrm{g}(\mathrm{x})-\mathrm{g}(\mathrm{y})+\frac{1}{\mathrm{r}}\langle y-z, z-x\rangle \geqslant 0, \forall \mathrm{y} \in \mathrm{C}\right\} .
$$

Therefore, by Theorem 3.1, we have the following.

Theorem 5.1. Let $\mathrm{H}_{1}, \mathrm{H}_{2}$ be two real Hilbert spaces and $\mathrm{C}, \mathrm{Q}$ be nonempty closed convex subsets of Hilbert spaces $\mathrm{H}_{1}$ and $\mathrm{H}_{2}$, respectively. Let $\mathrm{f}: \mathrm{C} \rightarrow \mathrm{R}$ and $\mathrm{g}: \mathrm{Q} \rightarrow \mathrm{R}$ be tow functions satisfying conditions (1) and (2). Let $A: \mathrm{H}_{1} \rightarrow \mathrm{H}_{2}$ be a bounded linear operator. Suppose that $\mathrm{O}(\mathrm{f}, \mathrm{A}) \neq \emptyset$. Let $\mathrm{x}_{0, \mathrm{i}} \in \mathrm{C}, \mathrm{i}=1,2,3, \cdots, N$, define 
sequences $\left\{x_{n, i}\right\}, i=1,2,3, \cdots, N$ and $\left\{\omega_{n}\right\}$ iteratively as follows:

$$
\left\{\begin{array}{l}
C_{1, i}=C, \quad i=1,2,3, \cdots, N, \\
u_{n, i}=O_{r_{n, i}}^{f}\left(I-\gamma_{i} A^{*}\left(I-O_{r_{n, i}}^{g}\right) A\right) x_{n, i}, \quad i=1,2,3, \cdots, N, \\
C_{n+1, i}=\left\{z \in C_{n, i}:\left\|u_{n, i}-z\right\| \leqslant\left\|x_{n, i}-z\right\|\right\}, \quad i=1,2,3, \cdots, N, \\
C_{n+1}=\bigcap_{i=1}^{N} C_{n+1, i}, \\
x_{n+1, i}=P_{C_{n+1}} x_{0, i}, \quad i=1,2,3, \cdots, N, \\
\omega_{n+1}=\sum_{i=1}^{N} \lambda_{i} x_{n+1, i}, \quad \sum_{i=1}^{N} \lambda_{i}=1, \quad \lambda_{i} \in[0,1]
\end{array}\right.
$$

for each $n \geqslant 1$, where $0<b \leqslant r_{n, i}<\infty, \gamma_{i} \in\left(0, \frac{1}{L}\right)$ for all $n \geqslant 1, i=1,2,3, \cdots, N$, $L$ is the spectral radius of the operator $\mathrm{A}^{*} \mathrm{~A}$ and $\mathrm{A}^{*}$ is the adjoint of $\mathrm{A}$. Then the following conclusions hold:

(1) $\left\{x_{n, i}\right\}$ converges strongly to $p_{i} \in O(f, A)$ for all $i=1,2,3, \cdots, N$;

(2) $\left\{\omega_{n}\right\}$ converges strongly to $\omega=\sum_{i=1}^{N} \lambda_{i} p_{i} \in O(f, A)$.

Proof. Take $T_{n}=I$ and $\alpha_{n}=0$ for all $n \geqslant 1$, where I denotes the identity operator. Taking $\alpha_{n}=0$, the desired result can be proved directly through Theorem 3.1.

Then, by Theorem 3.3, we have the following.

Theorem 5.2. Let $\mathrm{H}_{1}, \mathrm{H}_{2}$ be two real Hilbert spaces and $\mathrm{C}, \mathrm{Q}$ be nonempty closed convex subsets of Hilbert spaces $\mathrm{H}_{1}$ and $\mathrm{H}_{2}$, respectively. Let $\mathrm{f}: \mathrm{C} \rightarrow \mathrm{R}$ and $\mathrm{g}: \mathrm{Q} \rightarrow \mathrm{R}$ be two functions satisfying conditions (1) and (2). $A: \mathrm{H}_{1} \rightarrow \mathrm{H}_{2}$ be a bounded linear operator. Suppose that $\mathrm{O}(\mathrm{f}, \mathrm{A}) \neq \emptyset$. Let $\mathrm{x}_{0, \mathrm{i}} \in \mathrm{C}, \mathrm{i}=1,2,3, \cdots, \mathrm{N}$, define sequences $\left\{x_{n, i}\right\}, i=1,2,3, \cdots, N$ and $\left\{\omega_{n}\right\}$ iteratively as follows:

$$
\left\{\begin{array}{l}
\mathrm{C}_{1}=\mathrm{C} \\
\mathrm{u}_{\mathrm{n}}=\mathrm{O}_{\mathrm{r}_{n}}^{f}\left(\mathrm{I}-\gamma_{\mathrm{n}} A^{*}\left(\mathrm{I}-\mathrm{O}_{\mathrm{r}_{n}}^{\mathrm{g}}\right) A\right) w_{n}, \\
\mathrm{C}_{\mathrm{n}+1}=\left\{z \in \mathrm{C}_{n}:\left\|\mathrm{u}_{\mathrm{n}}-z\right\| \leqslant\left\|w_{n}-z\right\|\right\}, \\
x_{n, i}=\mathrm{P}_{\mathrm{C}_{n}} x_{0, i}, \quad i=1,2,3, \cdots, N, \\
\omega_{n}=\sum_{i=1}^{N} \lambda_{i} x_{n, i}, \quad \sum_{i=1}^{N} \lambda_{i}=1, \quad \lambda_{i} \in[0,1],
\end{array}\right.
$$

for each $n \geqslant 1$, where $0<b \leqslant r_{n}<\infty, \gamma_{n} \in\left(0, \frac{1}{L}\right)$ for all $n \geqslant 1, L$ is the spectral radius of the operator $A^{*} A$ and $\mathrm{A}^{*}$ is the adjoint of $\mathrm{A}$. Then the following conclusions hold:

(1) $\left\{x_{n, i}\right\}$ converges strongly to $p_{i} \in O(f, A)$ for all $i=1,2,3, \cdots, N$;

(2) $\left\{\omega_{n}\right\}$ converges strongly to $\omega=\sum_{i=1}^{N} \lambda_{i} p_{i} \in O(f, A)$.

Proof. Take $T_{n}=I$ and $\alpha_{n}=0$ for all $n \geqslant 1$, where I denotes the identity operator. Taking $\alpha_{n}=0$, the desired result can be proved directly through Theorem 3.3.

\section{Acknowledgment}

This project is supported by the major project of Hebei North University under grant No. ZD201304.

\section{References}

[1] R. P. Agarwal, J.-W. Chen, Y. J. Cho, Strong convergence theorems for equilibrium problems and weak Bregman relatively nonexpansive mappings in Banach spaces, J. Inequal. Appl., 2013 (2013), 16 pages. 1

[2] E. Blum, W. Oettli, From optimization and variational inequalities to equilibrium problems, Math. Student, 63 (1994), 123-145. 1, 2 
[3] A. Bnouhachem, Strong convergence algorithm for split equilibrium problems and hierarchical fixed point problems, Scientific World J., 2014 (2014), 12 pages. 1

[4] Y. Censor, A. Gibali, S. Reich, Algorithm for split variational inequality problems, Numer. Algorithms., 59 (2012) 301-323. 1, 4

[5] S.-S. Chang, H. W. Joseph Lee, C. K. Chan, A new method for solving equilibrium problem fixed point problem and variational inequality problem with application to optimization, Nonlinear Anal., 70 (2009), 3307-3319. 1

[6] S. Y. Cho, W.-L. Li, S. M. Kang, Convergence analysis of an iterative algorithm for monotone operators, J. Inequal. Appl., 2013 (2013), 14 pages. 1

[7] P. L. Combette, S. A. Hirstoaga, Equilibrium programming in Hilbert spaces, J. Nonlinear Convex Anal., 61 (2005), 117-136. 1, 1, 2.3, 2.4

[8] S. D. Flåm, A. S. Antipin, Equilibrium programming using proximal-like algorithms, Math. Programming, 78 (1997), 29-41. 1

[9] J.-Y. Guan, Y.-X. Tang, P.-C. Ma, Y.-C. Xu, Y.-F. Su, Non-convex hybrid algorithm for a family of countable quasi-Lipschitz mappings and application, Fixed Point Theory Appl., 2015 (2015), 11 pages. 1, 2.2

[10] Z.-H. He, The split equilibrium problem and its convergence algorithms, J. Inequal. Appl., 2012 (2012), 15 pages. 1

[11] H. Iiduka, W. Takahashi, Strong convergence theorems for nonexpansive mappings and inverse-strongly monotone mappings, Nonlinear Anal., 61 (2005), 341-350. 1, 2

[12] I. Inchan, Strong convergence theorems of modified Mann iteration methods for asymptotically nonexpansive mappings in Hilbert spaces, Int. J. Math. Anal. (Ruse), 2 (2008), 1135-1145. 1, 2

[13] P. Katchang, P. Kumam, A new iterative algorithm of solution for equilibrium problems, variational inequalities and fixed point problems in a Hilbert space, J. Appl. Math. Comput., 32 (2010), 19-38. 1

[14] K. Kazmi, S. H. Rizvi, terative approximation of a common solution of a split equilibrium problem, a variational inequality problem and a fixed point problem, J. Egyptian Math. Soc., 21 (2013), 44-51. 1

[15] J. K. Kim, Y. M. Nam, J. Y. Sim, Convergence theorems of implicit iterative sequences for a finite family of asymptotically quasi-nonxpansive type mappings, Nonlinear Anal., 71 (2009), e2839-e2848. 1, 2

[16] T.-H. Kim, H.-K. Xu, Strong convergence of modified Mann iterations for asymptotically nonexpansive mappings and semigroups, Nonlinear Anal., 64 (2006), 1140-1152. 2

[17] P.-K. Lin, K.-K. Tan, H.-K. Xu, Demiclosedness principle and asymptotic behavior for asymptotically nonexpansive mappings, Nonlinear Anal., 24 (1995), 929-946. 1

[18] H. Piri, R. Yavarimehr, Solving systems of monotone variational inequalities on fixed point sets of strictly pseudocontractive mappings, J. Nonlinear Funct. Anal., 2016 (2016), 18 pages. 1

[19] S. Plubtieng, R. Punpaeng, A general iterative method for equilibrium problems and fixed point problems in Hilbert spaces, J. Math. Anal. Appl., 336 (2007), 455-469. 1

[20] X.-L. Qin, M.-J. Shang, Y.-F. Su, A general iterative method for equilibrium problems and fixed point problems in Hilbert spaces, Nonlinear Anal., 69 (2008), 3897-3909. 1

[21] A. Tada, W. Takahashi, Weak and strong convergence theorems for a nonexpansive mapping and an equilibrium problem, J. Optim. Theory Appl., 113 (2007), 359-370. 1

[22] S. Takahashi, W. Takahashi, Viscosity approximation methods for equilibrium problems and fixed point problems in Hilbert spaces, J. Math. Anal. Appl., 331 (2007), 506-515.

[23] S. Takahashi, W. Takahashi, Strong convergence theorem for a generalized equilibrium problem and a nonexpansive mapping in a Hilbert space, Nonlinear Anal., 69 (2008), 1025-1033. 1

[24] U. Witthayarat, A. A. N. Abdou, Y. J. Cho, Shrinking projection methods for solving split equilibrium problems and fixed point problems for asymptotically nonexpansive mappings in Hilbert spaces, Fixed Point Theory Appl., 2015 (2015), 14 pages. 1 